\title{
On minimal surfaces bounded by two convex curves in parallel planes
}

Martin Traizet

\begin{abstract}
We prove that a compact minimal surface bounded by two closed convex curves in parallel planes close enough to each other must be topologically an annulus.
\end{abstract}

Mathematics Subject Classification (2000). 53A10.

Keywords. Minimal surface with boundary, Robin function, concentration of curvature.

\section{Introduction}

Let $\Gamma_{1}$ and $\Gamma_{2}$ be two closed convex curves in parallel planes in euclidean space, and let $M$ be a minimal annulus with boundary $\Gamma_{1}$ and $\Gamma_{2}$. In a celebrated paper [13], B. Shiffman proved that $M$ is foliated by convex curves in planes parallel to the planes of $\Gamma_{1}$ and $\Gamma_{2}$. Moreover, if $\Gamma_{1}$ and $\Gamma_{2}$ are circles, then $M$ is foliated by circles in parallel planes, and is therefore a piece of a catenoid or a Riemann minimal example.

It is natural to ask whether one can relax the hypothesis that $M$ is an annulus, or if other topological types are possible:

Can two convex curves in parallel planes bound a compact minimal surface of genus $\geq 1$ ?

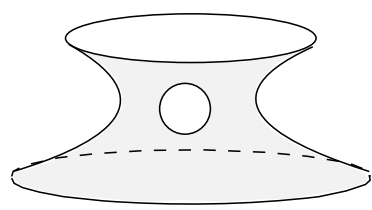

W. Meeks has conjectured that the answer to this question is no. Here is what is known about this conjecture. Without loss of generality we may assume that $\Gamma_{1}$ and $\Gamma_{2}$ are in horizontal planes. R. Schoen [12] has proven that the conjecture is true (so the answer to the question is no) if $\Gamma_{1}$ and $\Gamma_{2}$ are both symmetric with respect to the vertical planes $x_{1}=0$ and $x_{2}=0$, using the Alexandrov moving plane technique. 
A. Ros [11] has proven that the conjecture is true if $\Gamma_{2}$ is a vertical translate of $\Gamma_{1}$, using the Lopez-Ros deformation.

Even in the case of two circles with different axes, the conjecture seems to be open. Also using the bridge principle, one can construct examples of non-convex curves in parallel planes bounding a minimal surface of genus one.

In this paper, we study this problem in the case of two parallel planes close to each other. The question can be formulated more precisely as follows: let $\gamma_{1}$ and $\gamma_{2}$ be two convex curves in the horizontal plane $x_{3}=0$.

Is it true that if $T$ is a small enough vertical translation, then $\gamma_{1} \cup T\left(\gamma_{2}\right)$ does not bound any minimal surface of genus $k \geq 1$ ?

How small $T$ must be should depend in some way on the given curves $\gamma_{1}$ and $\gamma_{2}$, because of the invariance by scaling of the minimal surface equation. The main result of the paper is the following

Theorem 1. Let $\gamma_{1}$ and $\gamma_{2}$ be two smooth convex Jordan curves in the horizontal plane $x_{3}=0$, bounding respectively the convex domains $\Omega_{1}$ and $\Omega_{2}$. Fix some integer $k \geq 0$. Let $\left(M_{n}\right)_{n}$ be a sequence of compact, connected minimal surfaces of genus $k$ with boundary $\gamma_{1}$ and $T_{n}\left(\gamma_{2}\right)$, where $\left(T_{n}\right)_{n}$ is a sequence of vertical translations. If $k=0$, further assume that $M_{n}$ is not the stable annulus.

(1) (Compactness) If $T_{n} \rightarrow T \neq 0$, then a subsequence of $\left(M_{n}\right)_{n}$ converges smoothly to a compact minimal surface of genus $k$ bounded by $\gamma_{1}$ and $T\left(\gamma_{2}\right)$.

(2) (Concentration) If $T_{n} \rightarrow 0$, then there exists $k+1$ distinct points $p_{1}, \ldots, p_{k+1}$ in $\Omega_{1} \cap \Omega_{2}$ and a subsequence, still denoted $\left(M_{n}\right)_{n}$, such that the curvature of $\left(M_{n}\right)_{n}$ concentrates at $p_{1}, \ldots, p_{k+1}$, in the following sense: for any small $\rho>0$ it holds that

$$
\begin{gathered}
\lim _{n \rightarrow \infty} C\left(M_{n} \cap B\left(p_{i}, \rho\right)\right)=4 \pi \text { for all } i, \\
\lim _{n \rightarrow \infty} C\left(M_{n} \backslash \bigcup_{i=1}^{k+1} B\left(p_{i}, \rho\right)\right)=0,
\end{gathered}
$$

where $B(p, \rho)$ denotes the euclidean ball and $C(U)=\int_{U}|K| d A$ denotes the total curvature of $U$. Moreover, the configuration $p_{1}, \ldots, p_{k+1}$ is balanced, in an electrostatic sense which we explain in the next section.

We will see that near a point of concentration, the surface looks in fact like a small catenoid, which explains the $4 \pi$ mass of curvature.

In Section 2.3, we will prove that there are no balanced configurations in the genus one case $(k=1)$, so $T_{n} \rightarrow 0$ is impossible in this case. Hence, there exists $\varepsilon>0$ (depending on $\gamma_{1}$ and $\left.\gamma_{2}\right)$ such that if $\|T\|<\varepsilon, \gamma_{1} \cup T\left(\gamma_{2}\right)$ bounds no minimal 
surface of genus one. We will also give several partial results in the higher genus case, under various assumptions.

It is of course desirable to know how $\varepsilon$ depends on $\gamma_{1}$ and $\gamma_{2}$. For this, one has to allow the curves $\gamma_{1}$ and $\gamma_{2}$ to depend on $n$. We will prove a more general result in this case, see Theorem 2.

Remark 1. If $\gamma_{1} \cup T\left(\gamma_{2}\right)$ bounds a (connected) compact minimal surface $M$, then $\Omega_{1} \cap \Omega_{2}$ cannot be empty. Also $M$ is embedded, see Proposition 3 for these two facts.

Aknowledgements. I had interesting discussions about this problem with L. Hauswirth, D. Hoffman, L. Mazet and M. Wolf. I am especially grateful to L. Mazet for proving that there are no balanced configurations in the case $k \geq 1, \Omega_{1}=\Omega_{2}$, see Proposition 2. I would like to thank R. Mazzeo for pointing out the fact that the Robin function of a convex domain is convex, which plays an important role in Section 2. Finally, I would like to thank the referee for a very careful reading of the paper, finding two errors, and suggesting reference [5].

\section{Balanced configurations}

Let $\Omega_{1}$ and $\Omega_{2}$ be two bounded domains in the plane with non-empty intersection. Let $G_{i, p}(z)$ denotes the Green function of $\Omega_{i}$. Recall that $G_{i, p}(z)$ is harmonic in $\Omega_{i} \backslash\{p\}$ with zero boundary value and a logarithmic singularity at $p$. One can write

$$
G_{i, p}(z)=\log |z-p|+H_{i, p}(z),
$$

where the regular part $H_{i, p}(z)$ is harmonic in $\Omega_{i}$. It is known that $G_{i, p}(z)$ is a symmetric function of $(z, p)$.

Given $k+1$ distinct points $\left\{p_{1}, \ldots, p_{k+1}\right\}$ in $\Omega_{1} \cap \Omega_{2}$, let us define forces by

$$
F_{i}=\nabla H_{1, p_{i}}\left(p_{i}\right)+\nabla H_{2, p_{i}}\left(p_{i}\right)+\sum_{j \neq i}\left(\nabla G_{1, p_{j}}\left(p_{i}\right)+\nabla G_{2, p_{j}}\left(p_{i}\right)\right) \text {. }
$$

Definition 1. We say the configuration $\left\{p_{1}, \ldots, p_{k+1}\right\}$ is balanced if $F_{i}=0$ for $i=1, \ldots, k+1$.

When $\Omega_{1}=\Omega_{2}=\Omega$, one can interpret $F_{i}$ as 2-dimensional electrostatic forces. The physical model is the following: we have a 2 -dimensional vacuum chamber $\Omega$, whose boundary is made of a conductor metal. We put inside some unit positive charges at $p_{1} \ldots, p_{k+1}$. These charges induce a continuous charge on the boundary. Then $F_{i}$ is the force resulting of the interaction of $p_{i}$ with the other particles and with the boundary. 
Conjecture 1. If $\Omega_{1}$ and $\Omega_{2}$ are convex domains and $k \geq 1$, there are no balanced configuration with $k+1$ points.

We will prove that the conjecture is true in the case $k=1$, and give some partial results in the case $k \geq 2$. If we relax the convexity condition, then balanced configurations are possible. We will see an example in Section 2.5.

2.1. Facts about the Green function of a convex domain. In this section we collect several results about the Green function $G_{p}(z)$ of a bounded, convex domain $\Omega$. We write $G_{p}(z)=\log |z-p|+H_{p}(z)$, where $H_{p}(z)$ is the regular part of the Green function. The Robin function of $\Omega$ is defined by

$$
\operatorname{Rob}(z)=H_{z}(z) .
$$

The critical points of the Robin function are called the harmonic centers of $\Omega$. Since the Robin function goes to $+\infty$ on the boundary, any bounded domain has at least one harmonic center (a minimum). A very useful fact is the following:

The Robin function of a convex domain is convex.

This has been proven by various authors, see [2] and the references therein. The referee pointed out that in fact the Robin function of a bounded convex domain is strictly convex, see [5]. Therefore, a bounded convex domain has a unique harmonic center.

If $f: \mathbb{D} \rightarrow \Omega$ is a conformal representation of a domain $\Omega$ on the unit disk, one can compute the Green function of $\Omega$, its regular part and the Robin function in term of $f$ :

$$
\begin{aligned}
G_{f(p)}(f(z)) & =\log |z-p|-\log |1-\bar{p} z|, \\
H_{f(p)}(f(z)) & =-\log \left|\frac{f(z)-f(p)}{z-p}\right|-\log |1-\bar{p} z|, \\
\operatorname{Rob}(f(z)) & =-\log \left|f^{\prime}(z)\right|-\log \left(1-|z|^{2}\right) .
\end{aligned}
$$

Another fact about the Green function of a convex domain which we will use is the following

Lemma 1. Let $\Omega$ be a convex domain. Then for any $p \in \Omega$, the level lines of $G_{p}$ are convex curves.

Proof. This is very likely well known, but I could not find a reference in the literature, so I provide a proof. Fix some point $p \in \Omega$. Let $f: \mathbb{D} \rightarrow \Omega$ be a conformal representation of $\Omega$ such that $f(0)=p$. Then $G_{p}(f(z))=\log |z|$, so $f$ sends the 
circles centered at the origin to the level lines of $G_{p}$. Fix some $r \in(0,1)$ and let $\gamma_{r}(t)=f\left(r e^{\mathrm{i} t}\right)$. The image of $\gamma_{r}$ is convex if $\arg \gamma_{r}^{\prime}(t)$ is increasing. We have

$$
\begin{aligned}
\left(\arg \gamma_{r}^{\prime}(t)\right)^{\prime} & =\left(\operatorname{Im} \log \gamma_{r}^{\prime}(t)\right)^{\prime} \\
& =\operatorname{Im}\left(\frac{\gamma^{\prime \prime}(t)}{\gamma^{\prime}(t)}\right) \\
& =\operatorname{Im}\left(\mathrm{i} \frac{f^{\prime \prime}\left(r e^{\mathrm{i} t}\right)}{f^{\prime}\left(r e^{\mathrm{i} t}\right)} r e^{\mathrm{i} t}\right)+1:=g\left(r e^{\mathrm{i} t}\right),
\end{aligned}
$$

where the function $g$ is harmonic in $\mathbb{D}$, since $f^{\prime}$ does not vanish. When $r=1$, $\arg \gamma_{1}^{\prime}(t)$ is increasing because $\Omega$ is convex. Hence $g$ is non-negative on the unit circle. By the maximum principle, $g$ is positive in the disk, so the image of $\gamma_{r}$ is strictly convex if $r<1$.

2.2. Genus zero. In this section we discuss the case $k=0$, so there is only one point $p_{1}$. We write $\operatorname{Rob}_{i}(z)$ for the Robin function of $\Omega_{i}$. By symmetry of the Green function, $\nabla \operatorname{Rob}_{i}(z)=2 \nabla H_{i, z}(z)$, so

$$
F_{1}=\frac{1}{2}\left(\nabla \operatorname{Rob}_{1}\left(p_{1}\right)+\nabla \operatorname{Rob}_{2}\left(p_{1}\right)\right) .
$$

The configuration is balanced if $p_{1}$ is a critical point of $\operatorname{Rob}_{1}+\operatorname{Rob}_{2}$. Now the function $\operatorname{Rob}_{1}+\operatorname{Rob}_{2}$ is strictly convex on $\Omega_{1} \cap \Omega_{2}$, so it has a unique critical point (a minimum).

Returning to minimal surfaces, it is known that two convex curves in parallel planes bound at most two minimal annuli, one stable and one unstable [8]. Our result describes what happens to the unstable annulus when the distance between the planes goes to zero: the curvature concentrates at the minimum of the function $\operatorname{Rob}_{1}+\operatorname{Rob}_{2}$.

\subsection{Genus one}

Proposition 1. Let $k \geq 1$. If $\Omega_{1}$ and $\Omega_{2}$ are convex, then there are no balanced configurations with $k+1$ points, all on the same line $L$.

Proof. We may assume that the points $p_{1}, \ldots, p_{k+1}$ are in this order on $L$. Let $R=\frac{1}{2}\left(\operatorname{Rob}_{1}+\operatorname{Rob}_{2}\right)$. This is a convex function in $\Omega_{1} \cap \Omega_{2}$. Hence the maximum value of $R$ at the points $p_{1}, \ldots, p_{k+1}$ is either achieved at $p_{1}$ or $p_{k+1}$, let us say $p_{1}$. We have

$$
F_{1}=\nabla R\left(p_{1}\right)+\sum_{j>1} \nabla G_{1, p_{j}}\left(p_{1}\right)+\nabla G_{2, p_{j}}\left(p_{1}\right) .
$$

The point $p_{2}$ is inside the convex domain $R(z) \leq R\left(p_{1}\right)$ so $\left\langle\nabla R\left(p_{1}\right), \overrightarrow{p_{2} p_{1}}\right\rangle \geq 0$. Regarding the other terms, since $p_{j}$ lies inside the domain $G_{1, p_{j}}(z)<G_{1, p_{j}}\left(p_{1}\right)$ 
which is convex by Lemma 1 , we have $\left\langle\nabla G_{1, p_{j}}\left(p_{1}\right), \overrightarrow{p_{j} p_{1}}\right\rangle>0$, and a similar statement holds for the Green function of $\Omega_{2}$. Now all vectors ${\overrightarrow{p_{j}}}_{p_{1}}$ are proportional to $\overrightarrow{p_{2} p_{1}}$, with a positive coefficient, so we get $\left\langle F_{1}, \vec{p}_{2} \vec{p}_{1}\right\rangle>0$. Hence the configuration cannot be balanced.

In the case $k=1$, since two points are always on a line, there are no balanced configurations. This gives:

Corollary 1 (Genus one case). Given two smooth convex Jordan curves $\gamma_{1}$ and $\gamma_{2}$, there exists $\varepsilon>0$ (depending on $\gamma_{1}$ and $\gamma_{2}$ ), such that for any vertical translation $T$ with $\|T\|<\varepsilon, \gamma_{1} \cup T\left(\gamma_{2}\right)$ cannot bound any compact minimal surface of genus one.

2.4. Higher genus. In the case $k \geq 2$, we have a result under an additional symmetry assumption to ensure that the points $p_{1}, \ldots, p_{k+1}$ are on a line:

Corollary 2. Given two smooth convex Jordan curves $\gamma_{1}$ and $\gamma_{2}$, both symmetric with respect to a given line $L$, and some integer $k \geq 2$, there exists $\varepsilon$ (depending on $k, \gamma_{1}$ and $\left.\gamma_{2}\right)$ such that for any vertical translation $T$ with $\|T\|<\varepsilon, \gamma_{1} \cup T\left(\gamma_{2}\right)$ cannot bound any compact minimal surface of genus $k$.

Note that this corollary applies in particular to the interesting case of two circles.

Proof. Indeed, by a theorem of R. Schoen [12] (using Alexandrov's moving plane method), any minimal surface $M$ with boundary $\gamma_{1} \cup T\left(\gamma_{2}\right)$ will be symmetric with respect to the vertical plane $P$ through $L$. Moreover, the part of $M$ on each side of $P$ is a graph over $P$. Hence if we have a sequence of minimal surfaces $\left(M_{n}\right)_{n}$ of genus $k$, with boundary $\gamma_{1} \cup T_{n}\left(\gamma_{2}\right)$ with $T_{n} \rightarrow 0$, the curvature will concentrate at points $p_{1}, \ldots, p_{k+1}$, all on the line $L$ (this is because in a neighborhood of $p_{i}, M_{n}$ looks like a small catenoid, as we shall see). By Proposition 1, we get a contradiction.

Next we present a result which was discovered by L. Mazet.

Proposition 2. Assume that $\Omega_{1}$ and $\Omega_{2}$ have the same harmonic center. Then there are no balanced configurations with two or more points.

Note that the proposition applies in particular to the case where $\Omega_{1}=\Omega_{2}$. (Of course, in this particular case, the Meeks conjecture is known to be true by the work of A. Ros [11], so we do not get a new result, regarding minimal surfaces.)

Proof. Let $f_{i}: \mathbb{D} \rightarrow \Omega_{i}$ be a conformal representation. We transport the hyperbolic metric $2|d z| /\left(1-|z|^{2}\right)$ on the disk to get a hyperbolic metric $\lambda_{i}|d z|$ on $\Omega_{i}$. 
Explicitely,

$$
\lambda_{i}(z)=\frac{1}{\left|f_{i}^{\prime}\left(f_{i}^{-1}(z)\right)\right|\left(1-\left|f_{i}(z)\right|^{2}\right)}=2 \exp \left(\operatorname{Rob}_{i}(z)\right), \quad z \in \Omega_{i} .
$$

The hyperbolic distance $d_{\Omega_{i}}$ on $\Omega_{i}$ and the Green function are related by

$$
G_{i, p}(z)=\log \tanh \frac{d_{\Omega_{i}}(z, p)}{2} .
$$

This comes from the fact that the hyperbolic distance on the disk is given by

$$
d_{\mathbb{D}}(z, p)=2 \operatorname{arctanh}\left|\frac{z-p}{1-\bar{p} z}\right|
$$

Without loss of generality, we may assume that 0 is the harmonic center of $\Omega_{1}$ and $\Omega_{2}$, and that $\left|p_{1}\right| \geq\left|p_{j}\right|$ for all $j$ (so $p_{1} \neq 0$ ). Since $\operatorname{Rob}_{i}(0)<\operatorname{Rob}_{i}\left(p_{1}\right)$ and the Robin function is strictly convex, we have

$$
\left\langle\nabla \operatorname{Rob}_{i}\left(p_{1}\right), p_{1}\right\rangle>0
$$

Let us fix some indices $i=1,2$ and $j \geq 2$ and consider the geodesic $\gamma$ from $p_{j}$ to $p_{1}$ for the hyperbolic metric on $\Omega_{i}$. We know that this geodesic is minimizing. Let $\tau$ be the tangent vector to this geodesic at $p_{1}$. I claim that $\left\langle\tau, p_{1}\right\rangle \geq 0$. Indeed, if this is false, then since $\left|p_{j}\right| \leq\left|p_{1}\right|$, there exists a point $p \neq p_{1}$ on $\gamma$ such that $|p|=\left|p_{1}\right|$, and $|z|>\left|p_{1}\right|$ on the sub-arc $\gamma^{\prime}$ of $\gamma$ delimited by $p$ and $p_{1}$. Then consider the radial projection $\pi$ from $\gamma^{\prime}$ to the circle $C\left(0,\left|p_{1}\right|\right)$. By convexity, the Robin function, hence the conformal factor $\lambda_{i}$, is increasing on the segment $[0, z]$. Hence $\lambda_{i}(\pi(z))<\lambda_{i}(z)$. Since the projection makes euclidean length smaller, the hyperbolic length of the circular arc from $p$ to $p_{1}$ is smaller than the hyperbolic length of $\gamma^{\prime}$, which contradicts the fact that $\gamma$ is minimizing. Now the gradient of $G_{i, p_{j}}\left(p_{1}\right)$ is proportional to $\tau$, hence

$$
\left\langle\nabla G_{i, p_{j}}\left(p_{1}\right), p_{1}\right\rangle \geq 0
$$

This implies that $\left\langle F_{1}, p_{1}\right\rangle>0$, so the configuration cannot be balanced.

2.5. Explicit computations. When we have an explicit conformal representation $f: \mathbb{D} \rightarrow \Omega$ of a domain $\Omega$, we can compute explicitly the forces using the formulae in Section 2.1. It is convenient to identify $\mathbb{R}^{2}$ with $\mathbb{C}$ and use complex notations, so $\nabla=2 \frac{\partial}{\partial \bar{z}}$. The Robin function of the domain $\Omega$ satisfies

$$
\frac{\partial \operatorname{Rob}}{\partial z}(f(z)) \times f^{\prime}(z)=-\frac{f^{\prime \prime}(z)}{2 f^{\prime}(z)}+\frac{\bar{z}}{1-|z|^{2}} .
$$


Take $\Omega_{1}=\Omega_{2}=\Omega$ and consider a configuration $p_{1}, \ldots, p_{k+1} \in \Omega$. Writing $p_{i}=f\left(z_{i}\right), z_{i} \in \mathbb{D}$, the forces are given by

$$
\overline{F_{i}} \times f^{\prime}\left(z_{i}\right)=-\frac{f^{\prime \prime}\left(z_{i}\right)}{f^{\prime}\left(z_{i}\right)}+2 \sum_{j \neq i} \frac{1}{z_{i}-z_{j}}-2 \sum_{j} \frac{1}{z_{i}-\frac{1}{\overline{z_{j}}}} .
$$

We use these formula to provide counterexamples in the case of a non-convex domain. Consider for example

$$
f(z)=\frac{1}{z-a}+\frac{1}{z+a},
$$

where $a$ is some real number. Provided $a>1$ this is a conformal representation on the unit disk $\mathbb{D}$. When $a$ is close enough to 1 , the image $\Omega=f(\mathbb{D})$ is a non convex domain. Figure 1 shows this domain in the case $a=5 / 4$.

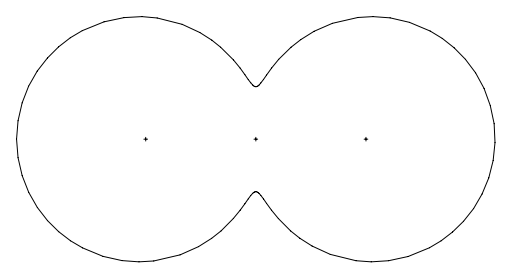

Figure 1. A non-convex domain admitting three harmonic centers.

Assume that $z$ is real. Then using the above formula, $f(z)$ is a harmonic center if $z^{3}\left(1-3 a^{2}\right)+z\left(3 a^{2}-a^{4}\right)=0$. Solving for $z$ and taking $f(z)$ gives three harmonic centers. These points are represented in Figure 1 when $a=5 / 4$. With a little more computations, it is possible to check that there are no other harmonic centers (namely, $z \notin \mathbb{R}$ ).

We can also compute a balanced configuration with two points, assuming the following symmetry: $z_{2}=-z_{1} \in \mathbb{R}$. The balancing condition boils down to a degree four equation, which gives two balanced configurations. One of them is represented in Figure 2, still in the case $a=5 / 4$. I do not know if there are other balanced configurations.

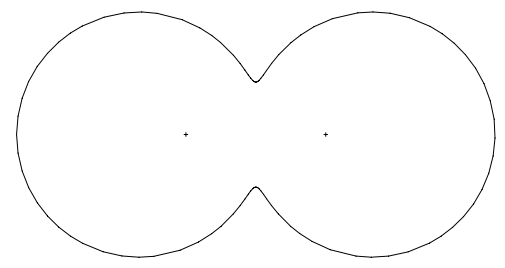

Figure 2. A balanced configuration with two points. 


\section{Proof of Theorem 1}

3.1. Preliminaries. Throughout the paper we use the following notations. $M$ is a compact embedded minimal surface of genus $k$, with boundary $\Gamma=\gamma_{1} \cup T\left(\gamma_{2}\right)$, where $T$ is a vertical translation of vector $(0,0, t)$ and $\gamma_{1}, \gamma_{2}$ are two convex Jordan curves in the horizontal plane $x_{3}=0 . \Omega_{1}$ and $\Omega_{2}$ denote the convex domains in the plane with boundary respectively $\gamma_{1}$ and $\gamma_{2}$. In case we have a sequence of minimal surfaces $\left(M_{n}\right)_{n}$, we label $\Gamma_{n}=\partial M_{n}, T_{n}=\left(0,0, t_{n}\right)$ and $\gamma_{i, n}=\partial \Omega_{i, n}$ the corresponding quantities. (The genus $k$ will always be fixed.)

The following proposition collects several elementary facts about minimal surfaces bounded by two convex curves in parallel planes.

Proposition 3. Let $M$ be a compact, connected minimal surface of genus $k$ bounded by two convex curves $\gamma_{1}$ and $T\left(\gamma_{2}\right)$. Then the following holds.

(1) The total curvature $C(M)$ of $M$ is at most $4 \pi(k+1)$.

(2) $M$ is embedded, and for any ball $B(p, R)$, the area of $M \cap B(p, R)$ is less than $2 \pi R^{2}$.

(3) $\Omega_{1} \cap \Omega_{2}$ is not empty.

(4) $M$ is contained in the intersection of the tubular neighborhood of radius $t$ of $\left(\Omega_{1} \cup \Omega_{2}\right) \times \mathbb{R}$ with the horizontal slab $0<x_{3}<t$.

(5) If $M$ is not a stable annulus, then for any disk $D$ of radius $\geq t$ included in $\Omega_{1} \cap \Omega_{2}, M$ intersects the vertical cylinder $D \times \mathbb{R}$.

Proof. By the Gauss-Bonnet formula,

$$
\int_{M} K+\int_{\partial M} \kappa_{g}=2 \pi \chi(M)=2 \pi(2-2 k-2) .
$$

This gives

$$
C(M)=-\int_{M} K=4 \pi k+\int_{\partial M} \kappa_{g} .
$$

Now it is well known that $\left|\kappa_{g}\right| \leq|\kappa|$, where $\kappa$ denotes the curvature of the boundary. As each $\gamma_{i}$ is a convex planar curve, $\int_{\gamma_{i}}|\kappa|=2 \pi$. This proves the first point. The second point is proven in [4], using the monotonicity formula for minimal surfaces with boundary. (Indeed, the boundary has total curvature $4 \pi$, and the density at $p$ of the cone with vertex $p$ generated by the boundary is less than 2 . The fact that the boundary is not connected is not a problem, see Section 6 in [4].)

Regarding point 3, let us assume by contradiction that $\Omega_{1} \cap \Omega_{2}=\emptyset$. Let $P$ be a vertical plane separating $\Omega_{1}$ and $\Omega_{2}$. Let $M^{\prime}$ be the symmetric of $M$ with respect to $P$. Let us translate $M^{\prime}$ horizontally in the direction of $P$. Since $M$ is connected, 
$M$ and $M^{\prime}$ will eventually intersect (maybe from the very beginning). First assume that $\bar{\Omega}_{1} \cap \bar{\Omega}_{2}=\emptyset$. Then the boundary of $M$ and $M^{\prime}$ never intersect, nor does the boundary of one intersect the interior of the other, since the interiors are in the slab delimited by the two horizontal planes. Hence at a last contact point, $M$ and $M^{\prime}$ are tangent, contradicting the maximum principle. If $\bar{\Omega}_{1}$ and $\bar{\Omega}_{2}$ intersect at some boundary point, one can slightly rotate $M^{\prime}$ about the horizontal line contained in $P$, so that the boundaries of $M$ and $M^{\prime}$ do not intersect. The convex hull property guarantees that the boundaries will not intersect the interiors, and the same argument applies.

To prove point 4 , let $C$ be a horizontal circle of radius $t$ in the horizontal plane $x_{3}=0$. There exists a catenoid $A$ bounded by $C \cup T(C)$. (The radius $t$ is not the smallest radius such that such a catenoid exists: the smallest value is about $0.754439 t$. The constants in the proposition are not optimal.) If the circle $C$ is disjoint from the convex hull of $\Omega_{1} \cap \Omega_{2}$ then $A$ does not intersect $M$. One can then slide $C$ horizontally. As long as $C$ remains disjoint from $\Omega_{1} \cup \Omega_{2}, A$ does not intersect $M$ by the maximum principle. This proves point 4 .

To prove point 5 , assume by contradiction that there exists a disk $D \subset \Omega_{1} \cap \Omega_{2}$ of radius $t$ such that $M$ does not intersect the vertical cylinder $D \times \mathbb{R}$. Let $C$ be the boundary of the disk $D$. Let us foliate each $\Omega_{i} \backslash D, i=1,2$, by convex curves $\gamma_{i, s}, s \in[0,1]$, so that $\gamma_{i, 0}=C$ and $\gamma_{i, 1}=\partial \Omega_{i}$. From the existence of a catenoid bounded by $C$ and $T(C)$ and Lemma 2.1 in [8], there exists, for each $s \in[0,1]$, a unique stable annulus $A_{s}$ bounded by $\gamma_{1, s} \cup T\left(\gamma_{2, s}\right)$. Moreover, as $A_{s}$ is stable and unique, it depends continuously on $s$ by standard results (namely, curvature estimates for stable minimal surfaces). By the maximum principle, $M$ is disjoint from $A_{s}$ for all $s \in[0,1)$. By point 1 of Lemma 2.1 in [8], $M$ is contained in the compact domain bounded by $A_{1}, \Omega_{1}$ and $T\left(\Omega_{2}\right)$. Hence $M=A_{1}$.

3.2. Main theorem. In this section we state a slightly more general result than Theorem 1, allowing the domains to depend on $n$.

Let $\left(\gamma_{1, n}\right)_{n}$ and $\left(\gamma_{2, n}\right)_{n}$ be two sequences of smooth convex Jordan curves in the plane, bounding the domains $\Omega_{1, n}$ and $\Omega_{2, n}$ respectively. Let $T_{n}$ be a sequence of vertical translations and $\left(M_{n}\right)_{n}$ be a sequence of minimal surfaces of fixed genus $k$ with boundary $\gamma_{1, n} \cup T_{n}\left(\gamma_{2, n}\right)$. If $k=0$, assume further that $M_{n}$ is not a stable minimal annulus. By point 3 of Proposition 3, each $\Omega_{1, n} \cap \Omega_{2, n}$ is non-empty. We assume that the in-radius of $\Omega_{1, n} \cap \Omega_{2, n}$ is greater than $r>0$, for some $r$ independent of $n$. We also assume that $\Omega_{1, n}$ and $\Omega_{2, n}$ are included in the disk $D(0, R)$ for some $R$ independent of $n$. Finally, we assume that the curvature of $\gamma_{1, n}$ and $\gamma_{2, n}$ is bounded by some constant independent of $n$. Passing to a subsequence, $\left(\gamma_{1, n}\right)_{n}$ and $\left(\gamma_{2, n}\right)_{n}$ converge to two convex Jordan curves $\gamma_{1}$ and $\gamma_{2}$, bounding respectively two convex domains $\Omega_{1}$ and $\Omega_{2}$ with non-empty intersection (thanks to the hypothesis on the in-radius). 
Theorem 2. In the above setup:

(1) (Compactness) If $T_{n} \rightarrow T \neq 0$, then a subsequence of $\left(M_{n}\right)_{n}$ converges smoothly to a compact minimal surface of genus $k$ bounded by $\gamma_{1}$ and $T\left(\gamma_{2}\right)$.

(2) (Concentration) If $T_{n} \rightarrow 0$, then there exists $k+1$ distinct points $p_{1}, \ldots, p_{k+1}$ in $\Omega_{1} \cap \Omega_{2}$ and a subsequence, still denoted $\left(M_{n}\right)_{n}$, such that the curvature of $\left(M_{n}\right)_{n}$ concentrates at $p_{1}, \ldots, p_{k+1}$, in the sense of Theorem 1 . Moreover, the configuration $p_{1}, \ldots, p_{k+1}$ is balanced, in the sense of Definition 1.

As a consequence, the constant $\varepsilon$ in Corollaries 1 and 2 depends on the following quantities: the genus $k$ of $M$, a bound on the curvature of $\gamma_{1}$ and $\gamma_{2}$, a bound on their diameter, and a lower bound on the in-radius of $\Omega_{1} \cap \Omega_{2}$.

Proof of point 1 of Theorem 2. By points 1 and 2 of Proposition 3, we have uniform area and total curvature estimates. By a standard compactness result, (namely by points 1, 2, 3 of Theorem 3 in [14]), there exists a subsequence of $\left(M_{n}\right)_{n}$, still denoted $\left(M_{n}\right)_{n}$, and a finite set $S$ in $\mathbb{R}^{3}$, such that $M_{n}$ converges on compact subsets of $\mathbb{R}^{3} \backslash S$ to an embedded minimal surface $M$ with boundary included in $\Gamma=\gamma_{1} \cup T\left(\gamma_{2}\right)$. Moreover, $M$ must be connected, else $M_{n}$ is not connected for $n$ large enough. If $M$ is flat, then its boundary lies in a plane, so $M$ is either in the plane $x_{3}=0$ or $x_{3}=t$. Since $t \neq 0$, this contradicts the fact than $\partial M_{n}=\gamma_{1, n} \cup T_{n}\left(\gamma_{2, n}\right)$. So $M$ is not flat. Let us see that the multiplicity of the limit $M_{n} \rightarrow M$ is one. The multiplicity is well defined and constant in each component of $M \backslash \Gamma$. Let $U$ be a component of $M \backslash \Gamma$ where the multiplicity $m$ is maximal, and assume that $m \geq 2$. Let $p$ be a point on $\partial U \subset \Gamma$. For small $r>0, B(p, r) \cap M_{n}$ has $m$ components. One of them meets $\partial M_{n}$. The others do not, and are graphs over $T_{p} M$ of functions which converge uniformly to the function which expresses locally $M$ as a graph over $T_{p} M$. Since $M_{n}$ lies in the horizontal slab $0 \leq x_{3} \leq t_{n}, T_{p} M$ must be horizontal. By the boundary maximum principle, since $M$ lies in the slab $0 \leq x_{3} \leq t, M$ is flat, a contradiction. Hence $M_{n} \rightarrow M$ with multiplicity one. By the proof of point 4 in Theorem 3 in [14], the singular set $S$ is empty. This proves point 1 of Theorem 2 .

The remaining of the paper is devoted to the proof of point 2 of Theorem 2 .

3.3. Limits under scaling. Let $\left(M_{n}\right)_{n}$ be a sequence of minimal surfaces as in the paragraph before Theorem 2 . Let $\left(h_{n}\right)_{n}$ be a sequence of homotheties of $\mathbb{R}^{3}$, with ratio diverging to $\infty$ as $n \rightarrow \infty$, and let $\tilde{M}_{n}=h_{n}\left(M_{n}\right)$. The goal of this section is to prove that the limit of $\left(\tilde{M}_{n}\right)_{n}$ is either flat or a catenoid.

Let $\tilde{\gamma}_{1, n}=h_{n}\left(\gamma_{1, n}\right), \tilde{\gamma}_{2, n}=h_{n}\left(T_{n}\left(\gamma_{2, n}\right)\right)$ and $\tilde{\Gamma}_{n}=\partial \tilde{M}_{n}=\tilde{\gamma}_{1, n} \cup \tilde{\gamma}_{2, n}$. Note that since the curvature of $\gamma_{i, n}$ is uniformly bounded, the curvature of $\tilde{\gamma}_{1, n}$ goes to zero as $n \rightarrow \infty$. If $\tilde{\gamma}_{i, n}$ has an accumulation point, then a subsequence of $\left(\tilde{\gamma}_{i, n}\right)_{n}$ converges on compact subsets of $\mathbb{R}^{3}$ to a horizontal line $L_{i}$. Hence passing 
to a subsequence, $\left(\widetilde{\Gamma}_{n}\right)_{n}$ converges to a set $\widetilde{\Gamma}$ which consists of zero, one or two horizontal lines. (When $\widetilde{\Gamma}=\emptyset$ this means that for any $R>0, \widetilde{\Gamma}_{n}$ is outside the ball $B(0, R)$ for $n$ large enough.)

By Theorem 3 in [14], there exists a finite set $S$ in $\mathbb{R}^{3}$ and a subsequence of $\left(\tilde{M}_{n}\right)_{n}$, still denoted the same, which converges on compact subsets of $\mathbb{R}^{3} \backslash(S \cup \widetilde{\Gamma})$ to a minimal surface $\widetilde{M}$ with boundary included in $\widetilde{\Gamma}$. Note that $\widetilde{M}$ can be disconnected.

Proposition 4. If $\tilde{M}$ has a non-flat component, then $S$ and $\widetilde{\Gamma}$ are empty and $\tilde{M}$ is a catenoid.

Proof. There are three cases, depending on whether $\widetilde{\Gamma}$ is empty, one line or two lines.

First case: $\widetilde{\Gamma}$ is empty. Then one component of $\widetilde{M}$ is a complete, embedded, nonflat minimal surface with finite total curvature. From the area estimate, point 2 of Proposition 3, it has at most two ends. Therefore it is a catenoid by the Theorem of R. Schoen [12]. By embeddedness $\tilde{M}$ has no other component. Since the catenoid is unstable, the multiplicity of the limit $\widetilde{M}_{n} \rightarrow \widetilde{M}$ is one by a standard argument (Proposition 4.2.1 in [9]), and the singular set $S$ is empty (Proposition 1.0.1 in [9], see also the end of the proof of Theorem 4.3.2).

Second case: $\widetilde{\Gamma}$ consists of one line $L$. Since $M_{n}$ lies in the slab $0 \leq x_{3} \leq t_{n}, \widetilde{M}$ lies in a half space bounded by the horizontal plane $\Pi$ containing $L$. Extending $\tilde{M}$ by reflection in $L$, we obtain a non-flat, embedded minimal surface in $\mathbb{R}^{3}$ with finite total curvature. By Theorem 2.2.1 in [9], a non-flat, embedded minimal surface of finite total curvature cannot intersect a plane along a line, so we get a contradiction. (This theorem uses the argument of J. Choe and M. Soret in [3].)

Third case: $\widetilde{\Gamma}$ consists of two lines $L_{1}$ and $L_{2}$. Since $M_{n}$ lies in the slab $0 \leq x_{3} \leq t_{n}$, $\widetilde{M}$ lies in the slab bounded by the horizontal planes containing $L_{1}$ and $L_{2}$. Since $\tilde{M}$ is non-flat, these two lines do not lie in the same horizontal plane. Hence we may assume that $L_{1}$ lies in the plane $x_{3}=0$ and $L_{2}$ lies in the plane $x_{3}=1$. The horizontal projections of $\tilde{\gamma}_{1, n}$ and $\tilde{\gamma}_{2, n}$ bound some convex domains $\widetilde{\Omega}_{1, n}$ and $\widetilde{\Omega}_{2, n}$, let $H_{i}=\lim \widetilde{\Omega}_{i, n}$. Then $H_{1}$ and $H_{2}$ are half planes, whose boundary lines are the horizontal projections of $L_{1}$ and $L_{2}$. By point 4 of Proposition 3 applied to $\widetilde{M}_{n}$ and letting $n \rightarrow \infty, \tilde{M}$ is inside the tubular neighborhood of radius one of $\left(H_{1} \cup H_{2}\right) \times \mathbb{R}$. By point 5 of the same proposition, for any disk $D$ of radius 1 contained in $H_{1} \cap H_{2}$, $\widetilde{M}$ intersects $D \times \mathbb{R}$. Let me call these two properties, respectively, property $A$ and property $B$. Roughly speaking, property $A$ means that the horizontal projection of $\widetilde{M}$ is contained in $H_{1} \cup H_{2}$, and property $B$ means that it contains $H_{1} \cap H_{2}$ (although not quite). As we shall see, properties $A$ and $B$ severely restrict the possibilities for the limit $\tilde{M}$.

Note that in case $L_{1}$ and $L_{2}$ are parallel, the boundaries of $\Omega_{1}$ and $\Omega_{2}$ are tangent at some point $p$. Since $\Omega_{1}$ and $\Omega_{2}$ are convex with non-empty intersection, they lie 
on the same side of this tangent line. Therefore, $H_{1} \subset H_{2}$ or $H_{2} \subset H_{1}$ (in other words, $H_{1} \cap H_{2}$ is not a strip).

Let $\theta$ be the angle between $L_{1}$ and $L_{2}$. Extending $\tilde{M}$ by reflection in $L_{1}$ and $L_{2}$, we obtain an embedded minimal surface $\widehat{M}$ in $\mathbb{R}^{3} / S_{2 \theta}$, where $S_{2 \theta}$ is a vertical screw motion of angle $2 \theta$ if $\theta \neq 0$, and a translation (maybe not vertical) in case $\theta=0$. Since $\widehat{M}$ has finite total curvature, a theorem of W. Meeks and H. Rosenberg [7] says that its ends are all simultaneously of type Scherk, helicoid or planar. We deal with each case separately.

First case: $\widehat{M}$ has Scherk type ends. Then the horizontal projection of $\tilde{M}$ stays at bounded distance from a finite set of half-lines, contradicting property $B$.

Second case: $\widehat{M}$ has helicoidal ends. In the case $\theta=0$, the period must be vertical, since $\tilde{M}$ lies in a horizontal slab. Each asymptotic half-helicoid intersects the horizontal plane $x_{3}=0$ along a half-line. Since $\hat{M}$ intersects the plane $x_{3}=0$ along the line $L_{1}$, it has precisely two helicoidal ends. Outside of a vertical cylinder, $\widetilde{M}$ has two components, each asymptotic to a piece of a helicoid, and having two half-lines on its boundary. Let us write $L_{1}=L_{1}^{\prime} \cup L_{1}^{\prime \prime}$ and $L_{2}=L_{2}^{\prime} \cup L_{2}^{\prime \prime}$, where $L_{1}^{\prime}, L_{2}^{\prime}$, $L_{1}^{\prime \prime}$ and $L_{2}^{\prime \prime}$ are half-lines defined as in Figure 3. Let $E_{1}$ and $E_{2}$ be the two pieces of helicoid that $\tilde{M}$ is asymptotic to, labeled so that $E_{1}$ has $L_{1}^{\prime}$ on its boundary. Note that by property $A$, none of them can make a full turn, and by property $B$, both cover $H_{1} \cap H_{2}$. If $E_{1}$ climbs from $L_{1}^{\prime}$ to $L_{2}^{\prime}$, then $E_{2}$ must climb from $L_{1}^{\prime \prime}$ to $L_{2}^{\prime \prime}$ (this can happen only in the non-parallel case). But then none of them covers $H_{1} \cap H_{2}$. Hence $E_{1}$ must climb from $L_{1}^{\prime}$ to $L_{2}^{\prime \prime}$, and $E_{2}$ from $L_{1}^{\prime \prime}$ to $L_{2}^{\prime}$. But then $E_{1}$ and $E_{2}$ intersect, which contradicts embeddedness. Therefore, $\widehat{M}$ cannot have helicoidal ends.
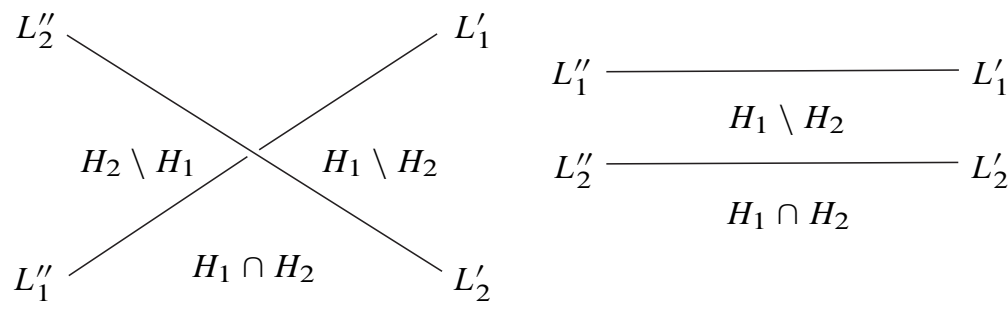

Figure 3. Definition of the four half-lines in the case where $L_{1}$ and $L_{2}$ are not parallel (left) or parallel (right).

Third case: $\widehat{M}$ has planar ends. Since $\tilde{M}$ lies in the slab $0 \leq x_{3} \leq 1$, the ends must be asymptotic to horizontal planes. By a theorem of Y. Choe and M. Soret [3], $\theta=0$, so the lines $L_{1}$ and $L_{2}$ are parallel. We may assume without loss of generality that $H_{1}$ is the half-plane $x_{1}>0$, then $H_{2}$ is the half-plane $x_{1}>a$ for some $a$. So $\tilde{M}$ is asymptotic to the half planes $x_{3}=0, x_{1}>0$ and $x_{3}=1, x_{1}>a$. Note that because 
of this, $\widehat{M}$ cannot be a Riemann minimal example: indeed the part of a Riemann minimal example between two consecutive horizontal lines is asymptotic to two half horizontal planes pointing into opposite directions.

To obtain a contradiction, we use the argument of Choe and Soret, as explained in [9]. We may assume that the stereographically projected Gauss map $g$ takes on the value 0 at the end at height $x_{3}=0$. Then by embeddedness, it must take on the value $\infty$ at the other end. Note that $g$ is real on $L_{1}$ and $L_{2}$.

By the boundary maximum principle for $\tilde{M}, g \neq 0, \infty$ on $L_{1}$, so $g$ has constant sign along $L_{1}$. Close to the end, $\tilde{M}$ lies in $x_{2}>0, x_{3}>0$, so we have $g>0$ on $L_{1}$. By a similar argument, $g$ is also positive on $L_{2}$.

Arguing as in [9], for $\varepsilon>0$ small enough, the intersection of $\tilde{M}$ with $0<x_{3}<\varepsilon$ is conformally an annulus $1<|z|<r$ for some $r>1$, with $x_{3}=0$ on $|z|=1$ and $x_{3}=\varepsilon$ on $|z|=r$. Since $x_{3}$ is harmonic, it follows that $x_{3}=\lambda \log |z|$ with $\lambda=\varepsilon / \log r$, and

$$
\phi_{3}=2 \frac{\partial x_{3}}{\partial z} d z=\lambda \frac{d z}{z} .
$$

(In [9], the authors claim that $\lambda=1$, but this is only the case after a suitable scaling of the surface.) If $\gamma$ is a closed curve on $\hat{M}$, let us define

$$
F(\gamma)=\mathrm{i} \int_{\gamma} \overline{g^{-1} \phi_{3}}=\mathrm{i} \int_{\gamma} g \phi_{3} .
$$

(These two integrals are equal because $\gamma$ is closed. $F(\gamma)$ represents the horizontal part of the flux along $\gamma$, seen as a complex number.) Let $\gamma_{s}$ be the curve $x_{3}=s$ on $\hat{M}$, oriented as a boundary of $x_{3}<s$. Then in the conformal representation, $\gamma_{\varepsilon}$ is the circle $|z|=r$, with the positive orientation. Since $g$ is holomorphic in $1 \leq|z| \leq r$,

$$
F\left(\gamma_{\varepsilon}\right)=\mathrm{i} \int_{|z|=r} g \phi_{3}=\mathrm{i} \int_{|z|=1} g \phi_{3}=\mathrm{i} \int_{\theta=0}^{2 \pi} g\left(e^{i \theta}\right) \lambda \mathrm{i} d \theta<0 .
$$

In the same way, we can represent conformally the intersection of $\tilde{M}$ with $1-\varepsilon<$ $x_{3}<1$ with an annulus $1<|z|<r$ for some other $r>1$, with $x_{3}=1-\lambda \log |z|$ and $\phi_{3}=-\lambda d z / z$. The level curve $\gamma_{1-\varepsilon}$ corresponds to the circle $|z|=r$, with the negative orientation:

$$
F\left(\gamma_{1-\varepsilon}\right)=-\mathrm{i} \int_{|z|=r} \overline{g^{-1} \phi_{3}}=-\mathrm{i} \int_{|z|=1} \overline{g^{-1} \phi_{3}}=-\mathrm{i} \int_{\theta=0}^{2 \pi}\left(g\left(e^{i \theta}\right)\right)^{-1} \lambda \mathrm{i} d \theta>0 .
$$

However, $F\left(\gamma_{\varepsilon}\right)=F\left(\gamma_{1-\varepsilon}\right)$ because the two curves are homologous. Hence we have a contradiction.

From Proposition 4 we get the following 
Proposition 5. Let $\left(M_{n}\right)_{n}$ be a sequence of minimal surfaces as in the paragraph before Theorem 2. Let $\left(h_{n}\right)_{n}$ be a sequence of homotheties of $\mathbb{R}^{3}$, and let $\widetilde{M}_{n}=$ $h_{n}\left(M_{n}\right)$. There exists a finite set $S$ and a subsequence of $\left(\tilde{M}_{n}\right)_{n}\left(\right.$ still denoted $\left.\left(\tilde{M}_{n}\right)_{n}\right)$ such that the curvature of $\left(\tilde{M}_{n}\right)_{n}$ is uniformly bounded on the compacts of $\mathbb{R}^{3} \backslash S$. Moreover, for any $p \in S$ and any $r>0$, it holds that

$$
\lim \sup C\left(\tilde{M}_{n} \cap B(p, r)\right) \geq 4 \pi \text {. }
$$

The point of this proposition is that for any point of concentration of curvature, the amount of total curvature which concentrates at this point is always at least $4 \pi$. This is well known for interior points of concentration (see for instance the proof of Theorem 3 in [14] or Theorem 4.3.1 in [9]), but wrong in general for boundary points of concentration. For example, if $\gamma_{1}$ and $\gamma_{2}$ are two convex curves which intersect at a finite number of points and $A_{n}$ is the stable annulus bounded by $\gamma_{1} \cup T_{n}\left(\gamma_{2}\right)$ with $T_{n} \rightarrow 0$, then the curvature concentrates at the intersection points of $\gamma_{1}$ and $\gamma_{2}$. The mass of curvature that concentrates at each point is equal to twice the angle between the curves at this point. (A blow-up would produce pieces of helicoids.)

This proposition can be proven exactly as Theorem 4.3.1 in [9], using a standard blowup argument. By Proposition 4, the only limits which can appear are catenoids, whence the $4 \pi$. We omit the details.

3.4. Weak limit. In this section, we adapt the weak compactness result of A. Ros [10] (in the case of complete embedded minimal surfaces of finite total curvature) to our case, namely when there is a boundary.

Proposition 6. Let $\left(M_{n}\right)_{n}$ be as in the paragraph before Theorem 2 and assume that $T_{n} \rightarrow 0$. There exists a subsequence, still denote $\left(M_{n}\right)_{n}$, and $k+1$ sequences of homotheties $\left(h_{i, n}\right)_{n}, 1 \leq i \leq k+1$, such that the following is true:

1) $h_{i, n}\left(M_{n}\right)$ converges smoothly on compact subsets of $\mathbb{R}^{3}$ to a vertical catenoid, with multiplicity one.

2) For any small $\varepsilon>0$, there exists $R>0$, independent of $n$, such that if we let $B_{i, n}=h_{i, n}^{-1}(B(0, R))$, then $C\left(M_{n} \backslash \bigcup_{i} B_{i, n}\right) \leq \varepsilon$.

3) For $n$ large enough, the balls $B_{i, n}$ are disjoint and $M_{n} \backslash \bigcup B_{i, n}$ has two components $U_{1, n}$ and $U_{2, n}$. Each $U_{j, n}$ is a graph over $\Omega_{j, n}$ minus $k+1$ small convex disks, for $j=1,2$.

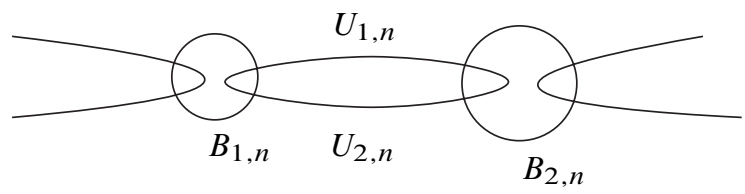

Figure 4. Weak limit, genus one. 
Remark 2. This proposition implies that $\lim C\left(M_{n}\right)=4 \pi(k+1)$.

Proof. We follow the main lines of the argument of A. Ros, adapted to the case of minimal surfaces with boundary. Passing to a subsequence, we may assume that $\lim C\left(M_{n}\right)$ exists. We write $\lim C\left(M_{n}\right)=4 \pi \ell+\alpha$ with $\ell \in \mathbb{N}$ and $0 \leq \alpha<4 \pi$. We first prove the following partial statement:

Claim 1. In the above setup, $\alpha=0$ and there exists $\ell$ sequences of homotheties $\left(h_{i, n}\right)_{n}$, such that for $1 \leq i \leq \ell,\left(h_{i, n}\left(M_{n}\right)\right)_{n}$ converges on compact subsets of $\mathbb{R}^{3}$ to a catenoid, with multiplicity one.

Proof. The idea of A. Ros to detect where the curvature concentrates is to look at balls $B$ such that $C\left(M_{n} \cap B\right)=2 \pi$, and to select the smallest such ball. It turns out that the value $2 \pi$ is not important for this argument: any fixed value $\mu \in(0,4 \pi)$ works fine. We choose $\mu$ as follows: if $\alpha=0$, we take $\mu=2 \pi$. If $\alpha>0$, we take $\mu=\alpha / 2$, and we want to get a contradiction. (In what follows, when we use the word "small", it means: "small compared to $\mu$ ". It is therefore important that $\mu$ is fixed once for all.)

First step. If $\ell=\alpha=0$, then the claim is trivially true. Else $\lim C\left(M_{n}\right)>\mu$, hence for $n$ large enough, the family of balls $B$ such that $C\left(M_{n} \cap B\right)=\mu$ is non-empty. Let $B_{1, n}^{\prime}$ be a ball of minimum radius in this family. Let $h_{1, n}$ be the homothety such that $h_{1, n}\left(B_{1, n}^{\prime}\right)=B(0,1)$ and let $\tilde{M}_{1, n}=h_{1, n}\left(M_{n}\right)$. Note that $C\left(\tilde{M}_{1, n} \cap B(0,1)\right)=\mu$ and that $B(0,1)$ is a smallest ball with this property. By Proposition 5, passing to a subsequence, there exists a finite set $S$ such that $\left(\widetilde{M}_{1, n}\right)_{n}$ converges to a minimal surface $\widetilde{M}_{1}$ on compact subsets of $\mathbb{R}^{3} \backslash S$. If $p \in S$, then by Proposition 5, $C\left(\tilde{M}_{1, n} \cap B\left(p, \frac{1}{2}\right)\right)>\mu$ for $n$ large enough. As this contradicts the choice of $B_{1, n}^{\prime}, S$ must be empty. Since $C\left(\tilde{M}_{1, n} \cap B(0,1)\right)=\mu>0, \tilde{M}_{1}$ cannot be flat. If the ratio of $h_{1, n}$ were bounded, then since $T_{n} \rightarrow 0, \widetilde{M}_{1}$ would be included in the horizontal plane, hence flat. Hence the ratio of $h_{1, n}$ is not bounded. By Proposition 4, $\tilde{M}_{1}$ is a catenoid and the multiplicity of the limit is one. Given $\varepsilon>0$, there exists $R_{1}>0$ such that $\left|C\left(\tilde{M}_{1} \cap B\left(0, R_{1}\right)\right)-4 \pi\right| \leq \varepsilon / 2$. From the smooth convergence of $\left(\widetilde{M}_{1, n}\right)_{n}$ to $\widetilde{M}_{1}$ on $B\left(0, R_{1}\right)$, we get $\left|C\left(\widetilde{M}_{1, n} \cap B\left(0, R_{1}\right)\right)-4 \pi\right| \leq \varepsilon$ for $n$ large enough. Let $B_{1, n}=h_{1, n}^{-1}\left(B\left(0, R_{1}\right)\right)$. Then $\left|C\left(M_{n} \cap B_{1, n}\right)-4 \pi\right| \leq \varepsilon$. In particular $\lim C\left(M_{n}\right) \geq 4 \pi$ and $\ell \geq 1$. This concludes the first step of the weak limit process.

Second step. If $\ell=1$ and $\alpha=0$ then we are done. Else $\lim C\left(M_{n}\right)>4 \pi+\mu$. By taking $\varepsilon$ small enough, for $n$ large enough, the family of balls $B$ such that $C\left(\left[M_{n} \backslash B_{1, n}\right] \cap B\right)=\mu$ is non-empty. Let $B_{2, n}^{\prime}$ be a ball of minimum radius in this family. Let $h_{2, n}$ be the homothety such that $h_{2, n}\left(B_{2, n}^{\prime}\right)=B(0,1)$ and let $\tilde{M}_{2, n}=h_{2, n}\left(M_{n}\right)$. Let $\widetilde{B}_{1, n}=h_{2, n}\left(B_{1, n}\right)$. By construction, the radius of $B_{1, n}^{\prime}$ is 
at most the radius of $B_{2, n}^{\prime}$. Hence $\widetilde{B}_{1, n}$ is a ball of radius at most $R_{1}$. Passing to a subsequence, the center of $\widetilde{B}_{1, n}$ either converges, or goes to infinity. We treat each case separately.

First case: The center of $\widetilde{B}_{1, n}$ diverges. Then we can argue as in the first step and conclude that $\left(\tilde{M}_{2, n}\right)_{n}$ converges on compact subsets of $\mathbb{R}^{3}$ to a catenoid $\tilde{M}_{2}$. There exists $R_{2}>0$ such that $\left|C\left(M_{n} \cap B_{2, n}\right)-4 \pi\right| \leq \varepsilon$, where $h_{2, n}\left(B_{2, n}\right)=B\left(0, R_{2}\right)$. For $n$ large enough, $B_{1, n}$ and $B_{2, n}$ are disjoint. Hence $\lim C\left(M_{n}\right) \geq 8 \pi$.

Second case: The center of $\widetilde{B}_{1, n}$ converges to a point $p$. In this case we want to obtain a contradiction. Passing to a subsequence, the radius of $\widetilde{B}_{1, n}$ has a limit $r$. If $r>0$, then from the convergence of $\tilde{M}_{1, n}$ to a catenoid, we obtain that $C\left(M_{n} \cap B_{2, n}^{\prime}\right) \leq \varepsilon$. This contradicts the definition of $B_{2, n}^{\prime}$. Hence $r=0$ and the sequence of balls $\left(\widetilde{B}_{1, n}\right)_{n}$ collapses into the point $p$. The sequence $\left(\tilde{M}_{2, n}\right)_{n}$ converges to $\tilde{M}_{2}$ with singular set $S$. Clearly $p \in S$, and in fact $S=\{p\}$, else we contradict the choice of $B_{2, n}^{\prime}$ as in the first step. Since $S$ is non-empty, all components of $\tilde{M}_{2}$ are flat by Proposition 4 . If $p \notin \overline{B(0,1)}$, then from the convergence of $\left(\tilde{M}_{2, n}\right)$ to a flat minimal surface on compact subsets of $\mathbb{R}^{3} \backslash\{p\}, C\left(\tilde{M}_{2, n} \cap B(0,1)\right) \rightarrow 0$. This contradicts the choice of $B_{2, n}^{\prime}$. Hence $p \in \overline{B(0,1)}$.

Fix a small $r>0$. For $n$ large enough, $\widetilde{B}_{1, n} \subset B(p, r)$. Let $\Sigma_{n}=\tilde{M}_{2, n} \cap$ $B(p, r) \backslash \widetilde{B}_{1, n}$. From the smooth convergence of $\left(\tilde{M}_{2, n}\right)_{n}$ to a flat limit on $B(0,1) \backslash$ $B(p, r)$, we have $\lim C\left(\tilde{M}_{2, n} \cap B(0,1) \backslash B(p, r)\right)=0$. Hence $\lim C\left(\Sigma_{n} \cap B(0,1)\right)=$ $\mu$. If $\lim C\left(\Sigma_{n}\right)>\mu$, then since $r<1$ we contradict the minimality of $B(0,1)$. Hence $\lim C\left(\Sigma_{n}\right)=\mu$.

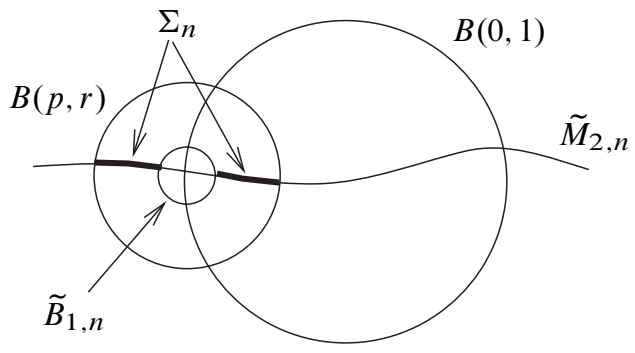

By looking at the Gauss image of $\Sigma_{n}$, we shall see that $\lim C\left(\Sigma_{n}\right)$ is a multiple of $4 \pi$, thus obtaining a contradiction. The boundary of $\Sigma_{n}$ is included in the union of the boundaries of $\widetilde{B}_{1, n}, B(p, r)$ and $\widetilde{M}_{2, n}$. On each component of $\partial \Sigma_{n} \cap \partial \widetilde{B}_{1, n}$, we have from the convergence to a catenoid that the Gauss map is close to a constant value (in fact arbitrarily close, by taking $R_{1}$ large enough). On each component of $\partial \Sigma_{n} \cap \partial B(p, r)$, the Gauss map is close to a a constant value: this follows from the convergence to a flat limit on compact subsets of $\mathbb{R}^{3} \backslash\{p\}$. Finally, we need 
to understand the Gauss map on $\partial \Sigma_{n} \cap \partial \tilde{M}_{2, n}$, in case this is not empty. On the boundary of $\tilde{M}_{2, n}$, the argument of the Gauss map is equal to the argument of the horizontal vector normal to the boundary. Since the curvature of the boundary of $\tilde{M}_{2, n}$ is bounded, the argument of the Gauss map on $\partial \widetilde{M}_{2, n} \cap B(p, r)$ is close to a constant value (arbitrarily close, by taking $r>0$ small enough). We conclude that the image by the Gauss map of each component of $\partial \Sigma_{n}$ is either a small disk, or a star-shaped curve bounding a small area on the sphere. Since the Gauss map is open, the image of $\Sigma_{n}$ has area close to a multiple of $4 \pi$. This contradicts the fact that $C\left(\Sigma_{n}\right)$ is close to $\mu$, and concludes the second step of our weak limit process.

We iterate this process $\ell$ times and produce $\ell$ sequences of homotheties $\left(h_{i, n}\right)_{n}$ and balls $\left(B_{i, n}\right)_{n}$ as wanted. Moreover, $\lim C\left(M_{n}\right) \geq 4 \pi \ell$. If $\alpha>0$, then by taking $\varepsilon>0$ small enough, we have that for $n$ large enough, the family of balls $B$ such that $C\left(\left[M_{n} \backslash \bigcup B_{i, n}\right] \cap B\right)=\mu$ is non-empty. So we can do one more step and conclude that $\lim C\left(M_{n}\right) \geq 4 \pi(\ell+1)$, a contradiction. Therefore $\alpha=0$. This proves the claim.

For $n$ large enough, the balls $B_{i, n}$ are disjoint, hence

$$
C\left(M_{n} \backslash \bigcup_{i=1}^{\ell} B_{i, n}\right) \leq \ell \varepsilon
$$

which proves point 2 of Proposition 6 (replacing $\varepsilon$ by $\varepsilon / \ell$ ).

Claim 2. The Gauss map converges to the vertical on each component of $\partial M_{n}$, in the following sense:

$$
\lim _{n \rightarrow \infty} \min _{x \in \partial M_{n}}\left|N_{3}(x)\right|=1
$$

Proof. We prove the claim for the bottom component of $\partial M_{n}$, the proof for the top component is similar. Let $x_{n}$ be a point on $\gamma_{1, n}$ such that $\left|N_{3}\left(x_{n}\right)\right|$ is minimum. Let $p_{i, n}$ be the center of $B_{i, n}$. Let $d_{n}=\min _{i} d\left(x_{n}, p_{i, n}\right)$. Passing to a subsequence, $\lim \frac{d_{n}}{t_{n}} \in[0, \infty]$ exists.

First case: $\lim \frac{d_{n}}{t_{n}}>0$ (possibly infinite). Let $h_{n}$ be the homothety of ratio $1 / t_{n}$ which maps $x_{n}$ to 0 . Let $\tilde{M}_{n}=h_{n}\left(M_{n}\right)$. By Proposition $5,\left(\tilde{M}_{n}\right)_{n}$ converges to a minimal surface $\tilde{M}$ with singular set $S$ (possibly empty). Moreover, $0 \notin S$, because else $\lim \frac{d_{n}}{t_{n}}=0$. Let $\widetilde{\Gamma}=\lim \partial \tilde{M}_{n}$, then $\widetilde{\Gamma}$ is a horizontal line $L_{1}$ through the origin, and possibly a line $L_{2}$ in the horizontal plane $x_{3}=1$. Since $\widetilde{\Gamma}$ is not empty, all components of $\tilde{M}$ are flat by Proposition 4 . If $\widetilde{\Gamma}=L_{1}$ or $L_{1}$ and $L_{2}$ are not parallel, then all components of $\tilde{M}$ must be planes or half-planes. Since $\tilde{M}_{n}$ lies in the horizontal slab $0 \leq x_{3} \leq 1$, all must be horizontal. Since $\tilde{M}_{n} \rightarrow \tilde{M}$ smoothly in a neighborhood of 0 , we conclude that $N_{3}\left(x_{n}\right)$ converges to a vertical vector. If $L_{1}$ 
and $L_{2}$ are parallel, then one component $U$ of $\tilde{M}$ might be the (non-horizontal) strip bounded by $L_{1}$ and $L_{2}$. By property B of the proof of Proposition 4 , there must be another component. This other component cannot be a half-plane, because $L_{1}$ and $L_{2}$ already bound $U$. Hence it must be a horizontal plane, but then we contradict property A of the proof of Proposition 4. We conclude that again all components of $\tilde{M}$ are horizontal planes and half-planes.

Second case: $\lim \frac{d_{n}}{t_{n}}=0$. In this case, let $h_{n}$ be the homothety of ratio $1 / d_{n}$ which maps $x_{n}$ to 0 . Let $\tilde{M}_{n}=h_{n}\left(M_{n}\right)$. By Proposition $4,\left(\tilde{M}_{n}\right)_{n}$ converges to a minimal surface $\tilde{M}$ with singular set $S \neq \emptyset$, and $d(0, S)=1$. Since $S \neq \emptyset$, all components of $\tilde{M}$ are flat by Proposition 4. Note that $\lim \partial \tilde{M}_{n}$ is a horizontal line $L$ containing the origin. Let us assume that there exists a component $U$ of $\tilde{M}$ which is not horizontal. Then since $\tilde{M}$ lies in the half space $x_{3} \geq 0, U$ must be a half-plane with boundary $L$, and its multiplicity is one, so $p \notin U$. The component of $\widetilde{M}$ containing $p$ must be a horizontal plane $x_{3}=a$, and its multiplicity is at least 2 . If $a>0$, then we contradict embeddedness. If $a=0$, then the density of $\tilde{M}$ at the origin is greater than or equal to $\frac{5}{2}$, so we contradict point 2 of Proposition 3 . Hence all components of $\tilde{M}$ are horizontal, so $N_{3}\left(x_{n}\right)$ converges to a vertical vector. This proves the claim.

It remains to prove that all catenoids are vertical, the third statement of Proposition 6 , and that $\ell=k+1$.

Let $U$ be a component of $M_{n} \backslash \bigcup B_{i, n}$. Since the balls $B_{i, n}$ do not intersect $\Gamma_{n}=\partial M_{n}$, each component of $\partial U$ is either a component of $\Gamma_{n}$, or a small circle included in some $\partial B_{i, n}$ (one of the two boundary components of the inside catenoid). By the previous claim or convergence to a catenoid, on each boundary component, the Gauss map is close to a constant. Since $C(U)$ is small, the Gauss map is close to a constant $a$ on $U$. Let $P=a^{\perp}$ and let $\pi: U \rightarrow P$ be the projection. Then $\pi$ is a local diffeomorphism so $\pi$ is open. Consider a component of $\partial U$ of the second type, namely a small circle $\gamma$ included in some $\partial B_{i, n}$. From the convergence to a catenoid, we can glue a disk along $\gamma$ in such a way that $\pi$ remains a local diffeomorphism. Perform this surgery for all such boundary circles $\gamma$ and call $\tilde{U}$ the result. Then $\pi: \tilde{U} \rightarrow P$ is a local diffeomorphism hence open. If $\partial U$ does not intersect $\Gamma_{n}$ then $\widetilde{U}$ is compact without boundary, but then $\pi: U \rightarrow P$ cannot be a local diffeomorphism. Hence $\partial U$ has a component equal to $\Gamma_{1, n}$ or $\Gamma_{2, n}$, so there are at most two such components $U$. Since the gauss map is close to a vertical constant on $\Gamma_{1, n}$ and $\Gamma_{2, n}$, we conclude that $P$ is the horizontal plane and all catenoids are vertical.

If $M_{n} \backslash \bigcup B_{i, n}$ has only one component $U$, then $\ell=0$. (Indeed, if $\ell \geq 1$, the Gauss map is close to a constant on the boundary of $B_{1, n} \cap M_{n}$, but this contradicts the convergence to a catenoid inside.) Since $\pi$ has no critical point on $M_{n}, M_{n}$ is an annulus. Since the Gauss map is close to a constant on $M_{n}, M_{n}$ is stable by the Barbosa do Carmo criterium. This is a contradiction since $M_{n}$ is not the stable annulus by hypothesis. 
Hence $M_{n} \backslash \bigcup B_{i, n}$ has precisely two components $U_{1, n}$ and $U_{2, n}$, with $\Gamma_{i, n} \subset$ $\partial U_{i, n}$. Gluing disks as above, the projection $\pi$ from $\widetilde{U}_{i, n}$ to the horizontal plane is open, and is one to one on $\partial \widetilde{U}_{i, n}=\Gamma_{i, n}$, so $\pi: \widetilde{U}_{i, n} \rightarrow \Omega_{i, n}$ is a diffeomorphism. This proves the third point of Proposition 6. Finally, the genus of $M_{n}$ is $\ell-1$, so $\ell=k+1$.

3.5. Flux. To make further progress we need the notion of flux. Let $\gamma$ be a curve on an oriented minimal surface $M$, and let $v$ be the co-normal along $\gamma$, chosen so that the basis $\left\{v, \gamma^{\prime}\right\}$ of the tangent plane is direct (so if $\gamma$ is the oriented boundary of some domain, $v$ is the exterior co-normal). The flux along $\gamma$ is the vector $\int_{\gamma} v d s$. This is a homology invariant vector. If we denote by $X^{*}=\left(X_{1}^{*}, X_{2}^{*}, X_{3}^{*}\right)$ the conjugate minimal immersion, then flux $(\gamma)=\int_{\gamma} d X^{*}$. If $M$ is the graph of a function $u(x, y)$, and is oriented by the upwards pointing normal, then one has the following formulae for the conjugate minimal immersion:

$$
\begin{aligned}
& d X_{1}^{*}=\frac{u_{x} u_{y} d x+\left[1+\left(u_{y}\right)^{2}\right] d y}{\sqrt{1+\left(u_{x}\right)^{2}+\left(u_{y}\right)^{2}}}, \\
& d X_{2}^{*}=\frac{-\left[1+\left(u_{x}\right)^{2}\right] d x-u_{x} u_{y} d y}{\sqrt{1+\left(u_{x}\right)^{2}+\left(u_{y}\right)^{2}}}, \\
& d X_{3}^{*}=\frac{u_{x} d y-u_{y} d x}{\sqrt{1+\left(u_{x}\right)^{2}+\left(u_{y}\right)^{2}}} .
\end{aligned}
$$

When $\nabla u$ is small, these formulae give the following expansions, with $z=x+\mathrm{i} y$ :

$$
\begin{aligned}
d X_{1}^{*}-\mathrm{i} d X_{2}^{*} & =\mathrm{i} d \bar{z}+2 \mathrm{i}\left(\frac{\partial u}{\partial z}\right)^{2} d z+o\left(|\nabla u|^{2}\right), \\
d X_{3}^{*} & =\operatorname{Im}\left(2 \frac{\partial u}{\partial z} d z\right)+o(|\nabla u|) .
\end{aligned}
$$

3.6. Limit rescaled graph. As we have seen, outside $k+1$ small balls, $M_{n}$ has two components $U_{1, n}$ and $U_{2, n}$. Each component $U_{i, n}$ is the graph over $\Omega_{i, n}$ minus small disks of a function which we call $u_{i, n}$. We have $u_{1, n}=0$ on $\partial \Omega_{1, n}$ and $u_{2, n}=t_{n}$ on $\partial \Omega_{2, n}$. In this section, we prove that after suitable scaling, these functions converge to explicit harmonic functions $u_{1}$ and $u_{2}$, each having $k+1$ logarithmic singularities.

Without loss of generality, we may assume (by changing the homotheties $h_{i, n}$ ) that all the limit catenoids are the standard catenoid $\cosh ^{2} x_{3}=x_{1}^{2}+x_{2}^{2}$. Let $\lambda_{i, n}$ be the ratio of $h_{i, n}$ and $\lambda_{n}=\min \lambda_{i, n}$. Passing to a subsequence, we may assume that $\lambda_{n}=\lambda_{i_{0}, n}$ for some index $i_{0}$. Passing again to a subsequence, the following limit exists:

$$
c_{i}=\lim _{n \rightarrow \infty} \frac{\lambda_{n}}{\lambda_{i, n}} \in[0,1] .
$$


Note that $c_{i_{0}}=1$, so at least one $c_{i}$ is non-zero. Let $p_{i, n} \in \mathbb{R}^{2}$ be the horizontal projection of the center of $B_{i, n}$. Passing to a subsequence, $p_{i}=\lim p_{i, n} \in \overline{\Omega_{1} \cap \Omega_{2}}$ exists. Note that at this point, we do not know that the points $p_{1}, \ldots, p_{k+1}$ are distinct.

Proposition 7. The following limits exist:

$$
\begin{gathered}
u_{1}:=\lim _{n \rightarrow \infty} \lambda_{n} u_{1, n}=-\sum_{i=1}^{k+1} c_{i} G_{1, p_{i}}, \\
u_{2}:=\lim _{n \rightarrow \infty} \lambda_{n}\left(u_{2, n}-t_{n}\right)(z)=\sum_{i=1}^{k+1} c_{i} G_{2, p_{i}},
\end{gathered}
$$

where $G_{i, p}$ denotes the Green function of $\Omega_{i}$. The convergence is the smooth convergence on compact subsets of $\Omega_{i} \backslash\left\{p_{1}, \ldots, p_{k+1}\right\}$.

Note that in this proposition, the points $p_{i}$ do not need to be distinct, and may also be on the boundary. If $p \in \partial \Omega_{i}, G_{i, p}$ should be understood as zero. Note that if $p$ converges to a boundary point $q$ of $\Omega_{i}$, then $G_{i, p}$ converges uniformly to 0 on compact subsets of $\overline{\Omega_{i}} \backslash\{q\}$ (this is easy to check by explicit formula for the disk, so is true for any bounded convex domain by conformal invariance of the Green function). This makes this definition natural.

Proof of the proposition. We orient $M_{n}$ so that the normal points up in $U_{1, n}$ and down on $U_{2, n}$. Let $\gamma_{1, i, n}$ and $\gamma_{2, i, n}$ denote the top and bottom boundary components of $M_{n} \cap B_{i, n}$ (oriented as boundaries). From the convergence to catenoids we have

$$
\lim _{n \rightarrow \infty} \lambda_{n} \operatorname{flux}\left(\gamma_{2, i, n}\right)=-\lim _{n \rightarrow \infty} \lambda_{n} \operatorname{flux}\left(\gamma_{1, i, n}\right)=\left(0,0,2 \pi c_{i}\right) .
$$

This gives

$$
\lim _{n \rightarrow \infty} \lambda_{n} \operatorname{flux}\left(\gamma_{1, n}\right)=-\lim _{n \rightarrow \infty} \lambda_{n} \operatorname{flux}\left(\gamma_{2, n}\right)=\sum_{i=1}^{k+1}\left(0,0,2 \pi c_{i}\right)
$$

Now the third coordinate of the co-normal $v$ has constant sign on each curve $\gamma_{1, i, n}$ and $\gamma_{2, i, n}$ (from the convergence to catenoids), and on $\gamma_{1, n}$ and $\gamma_{2, n}$ (from the convex hull property). Hence for $i=1,2$ we have the estimate

$$
\int_{\partial U_{i, n}} \lambda_{n}\left|d X_{3}^{*}\right| \leq C
$$


for some uniform constant $C$. Since the normal is close to be vertical on each $U_{i, n}$, we have $\sqrt{1+\left|\nabla u_{i, n}\right|^{2}} \leq 2$ for $n$ large enough, hence from equation (1), we have

$$
\int_{\partial U_{i, n}} \lambda_{n}\left|\nabla u_{i, n}\right| \leq 2 C
$$

From this integral estimate, we must conclude the convergence of a subsequence of $\left(\lambda_{n} u_{i, n}\right)_{n}$. If $u_{i, n}$ were harmonic, this would be quite elementary. So we make a conformal representation of $U_{i, n}$ onto a planar domain. Via this representation, $u_{i, n}$ becomes harmonic and we can conclude.

We shall only consider $u_{1, n}$, the proof for $u_{2, n}$ is entirely similar. By Koebe's theorem on uniformization of planar domains, there exists a conformal representation $f_{n}$ of $U_{1, n}$ onto the unit disk minus $k+1$ circular disks, such that $f_{n}$ maps $\gamma_{1, n}$ to the unit circle. Such a conformal representation is unique up to a Möbius transform of the disk. Let $\pi_{n}: U_{1, n} \rightarrow \Omega_{1, n}$ be the projection on the horizontal plane and let $\tilde{f}_{n}=$ $f_{n} \circ \pi_{n}^{-1}$. Using a Möbius transform of the disk, we may normalize $\tilde{f}_{n}$ by $\tilde{f}_{n}\left(z_{0}\right)=0$ and $\frac{\partial \tilde{f}_{n}}{\partial z}\left(z_{0}\right)>0$, where $z_{0}$ is a fixed point of $\Omega_{1}$, away from $p_{1}, \ldots, p_{k+1}$. Note that $\tilde{f}_{n}$ is defined on compact subsets of $\Omega_{1} \backslash\left\{p_{1}, \ldots, p_{k+1}\right\}$ for $n$ large enough, and is $\kappa_{n}$-quasi conformal with $\kappa_{n} \rightarrow 1$ as $n \rightarrow \infty$ (because $f_{n}$ is conformal and $\pi_{n}$ is $\kappa_{n}$-quasi conformal, since the Gauss map converges to a vertical vector). Since $\left(\tilde{f}_{n}\right)_{n}$ is bounded, by a standard normal family result ([6], Theorem 5.1, page 73), passing to a subsequence, $\left(\tilde{f}_{n}\right)_{n}$ converges on compact subsets of $\Omega_{1} \backslash\left\{p_{1}, \ldots, p_{k+1}\right\}$ to a 1-quasi conformal (hence holomorphic) function $f$. Moreover, $f\left(z_{0}\right)=0$ and $f^{\prime}\left(z_{0}\right) \geq 0$. By Riemann's theorem, $f$ extends holomorphically to $p_{1}, \ldots, p_{k+1}$. Let $q_{i}=f\left(p_{i}\right), i=1, \ldots, k+1$. By [6], Theorem 5.5, page 78, $f$ is either a diffeomorphism, or a constant function onto a boundary point, which is not possible since $f\left(z_{0}\right)=0$. Hence $f$ is the unique conformal representation of $\Omega_{1}$ onto the unit disk such that $f\left(z_{0}\right)=0, f^{\prime}\left(z_{0}\right)>0$. Since the limit is uniquely determined, the whole sequence $\left(\tilde{f}_{n}\right)_{n}$ converges to $f$.

Let $\Omega_{n}^{\prime}=f_{n}\left(U_{1, n}\right)$ and

$$
v_{n}=\lambda_{n} X_{3, n} \circ f_{n}^{-1}=\lambda_{n} u_{n} \circ \pi_{n} \circ f_{n}^{-1}, \quad \phi_{n}=\frac{\partial v_{n}}{\partial z},
$$

where $X_{3, n}: M_{n} \rightarrow \mathbb{R}$ denotes the third coordinate of the immersion. Since $M_{n}$ is minimal and $f_{n}$ is conformal, $v_{n}$ is a harmonic function so $\phi_{n}$ is a holomorphic function on $\Omega_{n}^{\prime}$. From equation (4) we have

$$
\int_{\partial \Omega_{n}^{\prime}}\left|\phi_{n}\right| \leq C
$$

Fix a small $\varepsilon>0$ and let $U_{\varepsilon}$ be the set of points in $D(0,1)$ which are at distance greater than $\varepsilon$ from $\partial D(0,1)$ and $q_{1}, \ldots, q_{k+1}$. Observe that for $n$ large enough, 
$U_{\varepsilon} \subset \Omega_{n}^{\prime}$ and if $z \in U_{\varepsilon}, d\left(z, \partial \Omega_{n}^{\prime}\right) \geq \varepsilon / 2$. By Cauchy's theorem we have, for $n$ large enough and $z \in U_{\varepsilon}$,

$$
\left|\phi_{n}(z)\right|=\frac{1}{2 \pi}\left|\int_{\partial \Omega_{n}^{\prime}} \frac{\phi_{n}(w)}{w-z} d w\right| \leq \frac{1}{2 \pi} \int_{\partial \Omega_{n}^{\prime}} \frac{\left|\phi_{n}\right|}{\varepsilon / 2} \leq \frac{C}{\pi \varepsilon} .
$$

Hence $\left(\phi_{n}\right)_{n}$ is bounded on $U_{\varepsilon}$. By the theorem on normal families, a subsequence of $\left(\phi_{n}\right)_{n}$ converges on compact subsets of $D(0,1) \backslash\left\{q_{1}, \ldots, q_{k+1}\right\}$ to a holomorphic function $\phi$. From the above estimate, $\phi$ has at most simple poles at each $q_{1}, \ldots, q_{k+1}$. Since $v_{n}=2 \operatorname{Re} \int \phi_{n}$, we obtain that $\left(v_{n}\right)$ converges to a harmonic function $v$ which has at most logarithmic singularities at $q_{1}, \ldots, q_{k+1}$ and vanishes on $\partial D(0,1)$. (To see that $v=0$ on the unit circle, we must ensure the convergence of $\left(\phi_{n}\right)_{n}$ on the boundary. This can be done as follows: since $v_{n}$ is zero on the unit circle, the 1 -form $\omega_{n}=\phi_{n} d z$ is pure imaginary on the unit circle. By the Schwartz reflection principle, one can extend the holomorphic one form $\omega_{n}$ by reflection in the circle namely, by $\sigma^{*} \omega_{n}=-\overline{\omega_{n}}$, where $\sigma(z)=1 / \bar{z}$. Fix some $r<1$ close to 1 . Then $\left(\omega_{n}\right)_{n}$ is bounded on the circles $|z|=r$ and $|z|=\frac{1}{r}$, so by the maximum principle, it is bounded in the annular region $r<|z|<\frac{1}{r}$. Hence, passing to a subsequence, the convergence holds up to $\partial D(0,1)$.)

Since $\lambda_{n} u_{n}=v_{n} \circ f_{n} \circ \pi_{n}^{-1},\left(\lambda_{n} u_{n}\right)_{n}$ converges to a harmonic function $u_{1}$ which is zero on $\partial \Omega_{1}$ and has at most logarithmic singularities at $p_{1}, \ldots, p_{k+1}$. By formula (3), the principal part of $u_{1}$ at $p_{i}$ is $-c_{i} \log \left|z-p_{i}\right|$. This proves the proposition.

3.7. The balancing condition. In this section, we compute the limit of the horizontal part of the flux, scaled by $\left(\lambda_{n}\right)^{2}$, on $\partial B_{i, n} \cap M_{n}=\gamma_{1, i, n} \cup \gamma_{2, i, n}$. Writing that this flux is zero will give the balancing condition. We assume that the configuration $p_{1}, \ldots, p_{k+1}$ is regular, in the following sense:

(1) the points $p_{1}, \ldots, p_{k+1}$ are distinct,

(2) $p_{i} \in \Omega_{1} \cap \Omega_{2}$ for all $i$.

A configuration is singular when several points are equal, or when some points are on the boundary of $\Omega_{1} \cap \Omega_{2}$. The case of singular configurations will be studied in Section 3.9. Let us define

$$
F(\gamma)=\operatorname{flux}_{1}(\gamma)-\mathrm{iflux}_{2}(\gamma)=\int_{\gamma} d X_{1}^{*}-\mathrm{i} d X_{2}^{*} .
$$

By formula (2), we have

$$
F\left(\gamma_{1, i, n}\right)=2 \mathrm{i} \int_{C\left(p_{i}, \varepsilon\right)}\left(\frac{\partial u_{1, n}}{\partial z}\right)^{2} d z+o\left(\left|\nabla u_{1, n}\right|^{2}\right),
$$




$$
\lim _{n \rightarrow \infty}\left(\lambda_{n}\right)^{2} F\left(\gamma_{1, i, n}\right)=2 \mathrm{i} \int_{C\left(p_{i}, \varepsilon\right)}\left(\frac{\partial u_{1}}{\partial z}\right)^{2} d z=-4 \pi \operatorname{Res}_{p_{i}}\left(\frac{\partial u_{1}}{\partial z}\right)^{2} .
$$

Now

$$
\frac{\partial u_{1}}{\partial z}=-\frac{c_{i}}{2\left(z-p_{i}\right)}-c_{i} \frac{\partial H_{1, p_{i}}}{\partial z}-\sum_{j \neq i} c_{j} \frac{\partial G_{1, p_{j}}}{\partial z}
$$

This gives, expanding the square and computing the residue,

$$
\lim _{n \rightarrow \infty}\left(\lambda_{n}\right)^{2} F\left(\gamma_{1, i, n}\right)=-4 \pi\left(c_{i}^{2} \frac{\partial H_{1, p_{i}}}{\partial z}\left(p_{i}\right)+\sum_{j \neq i} c_{i} c_{j} \frac{\partial G_{1, p_{j}}}{\partial z}\left(p_{i}\right)\right) .
$$

We have the same formula for $F\left(\gamma_{2, i, n}\right)$, replacing $H_{1, p_{i}}$ by $H_{2, p_{i}}$ and $G_{1, p_{j}}$ by $G_{2, p_{j}}$. (Regarding orientations: the normal points down in $U_{2, n}$, so there is a minus sign in front of the formulae for $d X^{*}$, and we must give $C\left(p_{i}, \varepsilon\right)$ the negative orientation, which gives another minus sign in front of the residue. These two minus signs compensate.) Since $\gamma_{1, i, n}+\gamma_{2, i, n}$ bounds $M_{n} \cap B_{i, n}$, the sum of the two fluxes is zero, so we obtain, for all $i=1, \ldots, k+1$,

$$
c_{i}^{2}\left(\frac{\partial H_{1, p_{i}}}{\partial z}\left(p_{i}\right)+\frac{\partial H_{2, p_{i}}}{\partial z}\left(p_{i}\right)\right)+\sum_{j \neq i} c_{i} c_{j}\left(\frac{\partial G_{1, p_{j}}}{\partial z}\left(p_{i}\right)+\frac{\partial G_{2, p_{j}}}{\partial z}\left(p_{i}\right)\right)=0 .
$$

This is not quite the balancing condition yet. We still must prove that all the $c_{i}$ are equal to one, which is the goal of the next section.

Remark 3. To prove that balanced configurations do not exist in Sections 2.3 and 2.4, we do not really need that all $c_{i}$ are equal to one: we could very well use the above balancing condition, provided that all $c_{i}$ are positive. However, the simplest way to prove that no $c_{i}$ vanishes seems to prove that all are in fact equal to one.

\subsection{Equal neck-sizes}

Proposition 8. Assume the configuration is non-singular (in the sense explained at the beginning of Section 3.7). Then all $c_{i}$ are equal to one.

Proof. For each neck, we use catenoidal barriers to estimate the height $t_{n}$ between the boundary curves as a function of $\lambda_{i, n}$. From this estimate we conclude that all $c_{i}=\lim \frac{\lambda_{i, n}}{\lambda_{n}}$ are equal.

Given $0<r<R$, let $\ell(r, R)$ be the part of the catenoid of waist radius $r$ defined by

$$
\sqrt{x_{1}^{2}+x_{2}^{2}}=r \cosh \left(x_{3} / r\right), \quad \sqrt{x_{1}^{2}+x_{2}^{2}}<R,
$$


so that $\mathcal{C}(r, R)$ is bounded by two horizontal circles of radius $R$ at height $\pm r \operatorname{argcosh} \frac{R}{r}$. Let $\ell^{+}(r, R)$ and $\ell^{-}(r, R)$ denote the upper half (in $x_{3}>0$ ) and lower half (in $\left.x_{3}<0\right)$ of $\varphi(r, R)$.

Let $p_{i, n} \in \mathbb{R}^{2}$ and $\eta_{i, n} \in\left(0, t_{n}\right)$ be respectively the horizontal projection and the third coordinate of the center of $B_{i, n}$, so $p_{i, n} \rightarrow p_{i}$ and $\eta_{i, n} \rightarrow 0$. Since the configuration is non-singular, there exists $\varepsilon>0$ such that for $n$ large enough, the disks $D\left(p_{i, n}, \varepsilon\right), i=1, \ldots, k+1$ are disjoint and inside $\Omega_{1, n} \cap \Omega_{2, n}$. From the convergence of $\left(\lambda_{n} u_{1, n}\right)_{n}$ to $u_{1}$ on compact subsets of $\Omega_{1} \backslash\left\{p_{1}, \ldots, p_{k+1}\right\}$, we have $\left|\lambda_{n} u_{1, n}\right| \leq C$ on the circles $C\left(p_{i, n}, \varepsilon\right)$ for some uniform constant $C$.

Upper bound for $\eta_{i, n}$. Fix some $\alpha>1$ close to one. Let $\Sigma_{i, n}$ be the part of $M_{n}$ inside the vertical cylinder $D\left(p_{i, n}, \varepsilon\right) \times\left(0, \eta_{i, n}\right)$. By convergence of $h_{i, n}\left(M_{n}\right)$ to a catenoid, the horizontal projection of the top component of $\partial \Sigma_{i, n}$ is a curve close to a circle of radius $1 / \lambda_{i, n}$, so it is inside the disk $D\left(p_{i, n}, \alpha / \lambda_{i, n}\right)$. (Here we assume, without loss of generality, that all limit catenoids are centered at the origin.) Consider the catenoid $\varrho\left(\alpha / \lambda_{i, n}, \varepsilon\right)$. Translate it horizontally so that its axis is the vertical line through $p_{i, n}$. Translate it vertically up so that it is disjoint from $\Sigma_{i, n}$, and then move it down.

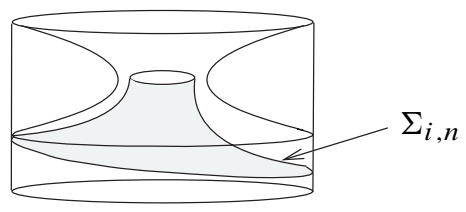

By the maximum principle, the first contact point will occur when the bottom circle touches the lower boundary component of $\partial \Sigma_{i, n}$, so its height will be at most $C / \lambda_{n}$. In this situation, the catenoid will be above $\Sigma_{i, n}$. The intersection of $\Sigma_{i, n}$ with $x_{3}=\eta_{i, n}-1 / \lambda_{i, n}$ is close to a circle of radius $\cosh (1) / \lambda_{i, n}$, which is greater than the waist radius of the catenoid, so $\eta_{i, n}-1 / \lambda_{i, n}$ must be less than the height of the waist of the catenoid. Using that $\operatorname{argcosh}(x) \leq \log (2 x)$ for $x \geq 1$, this gives the estimate

$$
\eta_{i, n} \leq \frac{C}{\lambda_{n}}+\frac{\alpha}{\lambda_{i, n}} \operatorname{argcosh} \frac{\varepsilon \lambda_{i, n}}{\alpha} \leq \frac{C^{\prime}}{\lambda_{n}}+\alpha \frac{\log \lambda_{i, n}}{\lambda_{i, n}}
$$

for some uniform constant $C^{\prime}$. By the same argument, we have the same upper bound for $t_{n}-\eta_{i, n}$. Adding the two estimates gives

$$
t_{n} \leq 2 \alpha \frac{\log \lambda_{i, n}}{\lambda_{i, n}}+\frac{2 C^{\prime}}{\lambda_{n}}
$$

Lower bound for $\eta_{i, n}$. Fix some $\beta<1$ close to one. Consider the lower half-catenoid $e^{-}\left(\beta / \lambda_{i, n}, \varepsilon\right)$. Translate it horizontally so that its axis is the vertical line through 
$p_{i, n}$. Translate it vertically down so that it is disjoint from $M_{n}$ and then up. By the maximum principle, the first contact point will occur when the bottom circle of the half-catenoid touches the boundary of $M_{n}$, and the part of $M_{n}$ inside the cylinder $D\left(p_{i, n}, \varepsilon\right) \times\left(0, t_{n}\right)$ will be above the catenoid. Using that $\operatorname{argcosh}(x) \geq \log (x)$ for $x \geq 1$, this gives the estimate

$$
\eta_{i, n} \geq \beta \frac{\log \lambda_{i, n}}{\lambda_{i, n}}-\frac{C^{\prime \prime}}{\lambda_{n}}
$$

for some uniform constant $C^{\prime \prime}$. By the same argument, we have the same lower bound for $t_{n}-\eta_{i, n}$. Adding the two estimate and taking $i=i_{0}$ (recall that $\lambda_{n}=\lambda_{i_{0}, n}$ by definition), we obtain

$$
t_{n} \geq 2 \beta \frac{\log \lambda_{n}}{\lambda_{n}}-\frac{2 C^{\prime \prime}}{\lambda_{n}} .
$$

Combining (6) and (5), we obtain

$$
\alpha \frac{\log \lambda_{i, n}}{\lambda_{i, n}} \geq \beta \frac{\log \lambda_{n}}{\lambda_{n}}-\frac{C^{\prime}+C^{\prime \prime}}{\lambda_{n}}
$$

which holds for any $\alpha>1$ and $\beta<1$, both close to one, and for $n$ large enough. From this we get

$$
\alpha \frac{\lambda_{n}}{\lambda_{i, n}} \log \left(\frac{\lambda_{i, n}}{\lambda_{n}}\right) \geq\left(\beta-\alpha \frac{\lambda_{n}}{\lambda_{i, n}}\right) \log \lambda_{n}-C^{\prime}-C^{\prime \prime} .
$$

The left hand side has a finite limit when $n \rightarrow \infty$, so $\beta-\alpha \frac{\lambda_{n}}{\lambda_{i, n}} \leq 0$ for $n$ large enough, else the right hand side goes to $+\infty$. This gives $c_{i} \geq \frac{\beta}{\alpha}$. The conclusion follows by letting $\alpha$ and $\beta$ go to one.

3.9. The singular case. Let us introduce some terminology. Let $p_{i}$ be a point of the configuration. If $p_{j} \neq p_{i}$ for all $j \neq i$ then we say that $p_{i}$ is a simple point, else that $p_{i}$ is a multiple point. If $p_{i}$ is not on the boundary of $\Omega_{1} \cap \Omega_{2}$ we say that $p_{i}$ is interior. If $c_{i}=0$, then we say that $p_{i}$ is evanescent. Evanescent points correspond to catenoidal necks which collapse too fast. Multiple points correspond to catenoidal necks which collapse to the same point. We want to prove that the configuration is non-singular, namely all points of the configuration are simple and interior.

If $\left(\varphi_{n}\right)_{n}$ is a sequence of homotheties of the plane with ratio $\mu_{n} \rightarrow \infty$, we define $\widetilde{\Omega}_{i, n}=\varphi_{n}\left(\Omega_{i, n}\right), \tilde{p}_{i, n}=\varphi_{n}\left(p_{i, n}\right)$ and $\tilde{u}_{i, n}=u_{i, n} \circ \varphi_{n}^{-1}$. Passing to a subsequence, $\tilde{p}_{i}=\lim \tilde{p}_{i, n}$ exists in $\mathbb{C} \cup\{\infty\}$, and $\widetilde{\Omega}_{i, n}$ converges to either a half-plane $H_{i}$ or the whole plane. We have the following generalization of Proposition 7 to this setup: 
Proposition 9. If $\lim \widetilde{\Omega}_{1, n}$ is a half-plane $H_{1}$, then

$$
\lim _{n \rightarrow \infty} \lambda_{n} \tilde{u}_{1, n}(z)=-\sum_{i=1}^{k+1} c_{i} \log \left|\frac{z-\tilde{p}_{i}}{z-\sigma_{1}\left(\tilde{p}_{i}\right)}\right|,
$$

where $\sigma_{1}$ denotes the symmetry with respect to the boundary line of $H_{1}$. If $\lim \widetilde{\Omega}_{1, n}$ is the whole plane, then

$$
\lim _{n \rightarrow \infty} \lambda_{n}\left(\tilde{u}_{1, n}(z)-\tilde{u}_{1, n}\left(z_{0}\right)\right)=-\sum_{i=1}^{k+1} c_{i} \log \left|\frac{z-\tilde{p}_{i}}{z_{0}-\tilde{p}_{i}}\right| .
$$

The convergence is on compact subsets of $H_{1}$ or $\mathbb{C}$ minus $\tilde{p}_{1}, \ldots, \tilde{p}_{k+1}$. In case $\tilde{p}_{i}=\infty$, the corresponding term in the above formulae should be understood as zero. A similar statement holds for $\tilde{u}_{2, n}$.

We start by proving:

Proposition 10. The points which are not evanescent are interior and simple amongst non-evanescent points (which simply means that they are distinct).

Proof. the proof is by contradiction. If this is not true, then by making blow-ups, we obtain a balanced configuration as before, with the domains $\Omega_{1}$ and $\Omega_{2}$ replaced by half-planes (in the case of a boundary point) or the whole plane (in the case of a multiple point). We obtain a contradiction by proving that balanced configuration are impossible in these cases. Note that forces won't see evanescent points, which is why we only get information about non-evanescent points. We will rule out evanescent points in the next proposition.

Without loss of generality, we may assume that the points which are not evanescent are $p_{1}, \ldots, p_{r}$, for some $r \geq 1$. Let

$\delta_{n}=\min \left(\left\{d\left(p_{i, n}, \partial\left(\Omega_{1, n} \cap \Omega_{2, n}\right)\right), 1 \leq i \leq r\right\} \cup\left\{d\left(p_{i, n}, p_{j, n}\right), 1 \leq i<j \leq r\right\}\right)$.

We want to prove that inf $\delta_{n}>0$. Assume by contradiction that inf $\delta_{n}=0$. Then we can find a subsequence such that $\lim \delta_{n}=0$. Passing to a subsequence, and maybe changing indices, $\delta_{n}$ is always equal to the distance of $p_{1, n}$ to the boundary or to $d\left(p_{1, n}, p_{2, n}\right)$. Let $\varphi_{n}$ be the homothety of ratio $\mu_{n}=1 / \delta_{n}$ in the plane which maps $p_{1, n}$ to the origin. Then passing to a subsequence and using the notations before Proposition 9, $\tilde{p}_{i}=\lim \tilde{p}_{i, n} \in \mathbb{C} \cup\{\infty\}$ and $\widetilde{\Omega}_{\ell}=\lim \widetilde{\Omega}_{\ell, n}$ exist, $\ell=1,2$. Moreover, $\tilde{p}_{1}=0$, and the points $\tilde{p}_{1}, \ldots, \tilde{p}_{r}$ are at distance at least one from each other and from the boundary. We may assume that the points $\tilde{p}_{i}$ which are finite are $\tilde{p}_{1}, \ldots, \tilde{p}_{s}$ for some $s \geq 1$. 
If $\widetilde{\Omega}_{\ell}=\mathbb{C}$ then arguing as in Section 3.7 and using Proposition 9, we have

$$
\lim _{n \rightarrow \infty} \frac{\lambda_{n}^{2}}{\mu_{n}^{2}} F\left(\gamma_{\ell, i, n}\right)=-4 \pi \operatorname{Res}_{\tilde{p}_{i}}\left(\frac{\partial}{\partial z} \sum_{j=1}^{s} c_{j} \log \left|z-\tilde{p}_{j}\right|\right)^{2}=-2 \pi \sum_{j \neq i} \frac{c_{i} c_{j}}{\tilde{p}_{i}-\tilde{p}_{j}} .
$$

If $\widetilde{\Omega}_{\ell}=H_{\ell}$ is a half-plane then we have $\left(\sigma_{\ell}(z)\right.$ denotes again the symmetry with respect to the boundary of $H_{\ell}$ )

$$
\begin{aligned}
\lim _{n \rightarrow \infty} \frac{\lambda_{n}^{2}}{\mu_{n}^{2}} F\left(\gamma_{\ell, i, n}\right) & =-4 \pi \operatorname{Res}_{\tilde{p}_{i}}\left(\frac{\partial}{\partial z} \sum_{j=1}^{s} c_{j} \log \left|z-\tilde{p}_{j}\right|-c_{j} \log \left|z-\sigma_{\ell}\left(\tilde{p}_{j}\right)\right|\right)^{2} \\
& =-2 \pi\left(\sum_{j \neq i} \frac{c_{i} c_{j}}{\tilde{p}_{i}-\tilde{p}_{j}}-\sum_{j=1}^{s} \frac{c_{i} c_{j}}{\tilde{p}_{i}-\sigma_{\ell}\left(\tilde{p}_{j}\right)}\right) .
\end{aligned}
$$

First case: Both $\widetilde{\Omega}_{1}$ and $\widetilde{\Omega}_{2}$ are the whole plane $\mathbb{C}$. Then necessarily $s \geq 2$, and the above formulae give the balancing formula:

$$
2 \sum_{j \neq i} \frac{c_{i} c_{j}}{\tilde{p}_{i}-\tilde{p}_{j}}=0 \text { for all } i \leq s .
$$

Since $s \geq 2$, it is straightforward to see that there are no balanced configurations $\tilde{p}_{1}, \ldots, \tilde{p}_{s}$. (Simply consider an extremal point, namely which is not in the convex hull of the others. The force on such a point cannot vanish.)

Second case: $\widetilde{\Omega}_{1}=H_{1}$ is a half-plane and $\widetilde{\Omega}_{2}$ is the whole plane. By rotation and translation, we may assume that $H_{1}$ is the half plane $\operatorname{Im}(z)>0$, so $\sigma_{1}(z)=\bar{z}$. The above formulae give the balancing condition:

$$
\sum_{j \neq i} \frac{c_{i} c_{j}}{\tilde{p}_{i}-\tilde{p}_{j}}+\sum_{j=1}^{s} \frac{-c_{i} c_{j}}{\tilde{p}_{i}-\overline{\tilde{p}_{j}}}=0 \quad \text { for all } i \leq s .
$$

If $\tilde{p}_{i}$ has the smallest imaginary part amongst $\tilde{p}_{1}, \ldots, \tilde{p}_{s}$, then all terms in the first sum have non-negative imaginary part, and all terms in the second have positive imaginary part, hence the force cannot be zero. The case where $\widetilde{\Omega}_{1}$ is the whole plane and $\widetilde{\Omega}_{2}$ is a half-plane is identical.

Third case: $\widetilde{\Omega}_{1}=H_{1}$ and $\widetilde{\Omega}_{2}=H_{2}$ are both half-planes. Note that $H_{1} \cap H_{2}$ cannot be a strip, because this would contradict the fact that $\Omega_{1, n} \cap \Omega_{2, n}$ contains a disk of fixed radius. So by translation and rotation we may assume that for $\ell=1,2, H_{\ell}$ is the half-plane $y>\tan \left(\alpha_{\ell}\right) x$ for some $\alpha_{\ell} \in\left(-\frac{\pi}{2}, \frac{\pi}{2}\right)$. We obtain the balancing condition:

$$
\sum_{\ell=1}^{2}\left(\frac{-c_{i}^{2}}{\tilde{p}_{i}-\sigma_{\ell}\left(\tilde{p}_{i}\right)}+\sum_{j \neq i}\left[\frac{c_{i} c_{j}}{\tilde{p}_{i}-\tilde{p}_{j}}-\frac{c_{i} c_{j}}{p_{i}-\sigma_{\ell}\left(\tilde{p}_{j}\right)}\right]\right)=0 \quad \text { for all } i \leq s .
$$


Fix some $\ell=1,2$, and write $H=H_{\ell}, \sigma=\sigma_{\ell}$. If $z, w$ are points in $H$ then we clearly have $\operatorname{Im}(\sigma(z))<\operatorname{Im}(z)$ and $|\sigma(w)-z|>|w-z|$. If $\operatorname{Im}(z) \leq \operatorname{Im}(\sigma(w))$ then we have

$$
\begin{aligned}
\operatorname{Im}\left(\frac{1}{z-w}-\frac{1}{z-\sigma(w)}\right) & =\frac{\operatorname{Im}(w-z)}{|z-w|^{2}}+\frac{\operatorname{Im}(z-\sigma(w))}{|z-\sigma(w)|^{2}} \\
& \geq \frac{\operatorname{Im}(w-z)}{|z-w|^{2}}+\frac{\operatorname{Im}(z-\sigma(w))}{|z-w|^{2}}=\frac{\operatorname{Im}(w-\sigma(w))}{|z-w|^{2}}>0 .
\end{aligned}
$$

If $\operatorname{Im}(\sigma(w))<\operatorname{Im}(z) \leq \operatorname{Im}(w)$ then the same conclusion holds (this time both terms are positive).

Now consider the point $\tilde{p}_{i}$ which has the smallest imaginary part. It follows from what we have just seen that the first term and all brackets in (7) have positive imaginary part. So the force on $\tilde{p}_{i}$ cannot vanish. This proves the proposition.

\section{Proposition 11. The configuration is non-singular.}

Proof. If we look at the proof of Proposition 8, we see that to get the upper bound for $\eta_{i, n}$, we only need that the point $p_{i}$ is simple, while for the lower bound of $\eta_{i, n}$, we only need that the point $p_{i}$ is interior. Since $c_{i_{0}}=1$, Proposition 10 says that $p_{i_{0}}$ is interior. Hence the lower bound for $t_{n}$, equation (6), holds.

Let $p_{i}$ be a point of the configuration. If $p_{i}$ is simple, then as we observed above, we can obtain an upper bound for $\eta_{i, n}$ and conclude that $c_{i}=1$ as in Section 3.8, so $p_{i}$ is interior by Proposition 10. Therefore, to prove the proposition, we only have to prove that all points are simple.

Assume by contradiction that there exists a multiple point. By changing indices, we may assume that $p_{1}=p_{2}=\cdots=p_{m}$ for some integer $m \geq 2$. Passing to a subsequence and changing indices, we may assume that $\lambda_{1, n}=\min \left\{\lambda_{i, n}: 1 \leq i \leq m\right\}$ for all $n$. Our first goal is to prove that $c_{1}>0$ by obtaining an upper bound for $\eta_{1, n}$. We estimate the height $\eta_{1, n}$ using an extremal length argument, which is more flexible than the use of a catenoidal barrier, although it gives a cruder result.

Let $\Gamma$ be a family of curves in the plane. The extremal length $\lambda(\Gamma)$ of $\Gamma$ is defined as follows (see Ahlfors' book [1]):

$$
\begin{gathered}
\lambda(\Gamma)=\sup _{\rho} \frac{L(\rho)^{2}}{A(\rho)} ; \\
L(\rho)=\inf _{\gamma \in \Gamma} L_{\gamma}(\rho), \quad L_{\gamma}(\rho)=\int_{\gamma} \rho|d z|, \quad A(\rho)=\iint \rho^{2} d x d y .
\end{gathered}
$$

Here $\rho$ is any measurable non-negative function in the plane, such that $A(\rho) \neq$ $0, \infty$. If $\Omega$ is an annulus and $\Gamma$ is the set of curves which connect its two boundary components, then $\lambda(\Gamma)$ is called the modulus of $\Omega$. The modulus is a conformal 
invariant and is monotonous, namely $\Omega \subset \Omega^{\prime} \Rightarrow \bmod (\Omega) \leq \bmod \left(\Omega^{\prime}\right)$. The modulus of the annulus $D(0, R) \backslash D(0, r)$ is $\frac{1}{2 \pi} \log \frac{R}{r}$.

There exists $\varepsilon>0$ such that all points of the configuration are either equal to $p_{1}$ or at distance greater than $2 \varepsilon$ from $p_{1}$. By Proposition 7 , we have $\left|\lambda_{n} u_{1, n}\right|<C$ on the circle $C\left(p_{1, n}, \varepsilon\right)$ for some uniform constant $C$. Let $a_{n}=\frac{C}{\lambda_{n}}$. Consider the subset of $\pi\left(U_{1, n}\right) \subset \Omega_{1, n}$ defined by $u_{1, n}>a_{n}$. Let $\Sigma_{1}$ the component which has $\pi\left(\gamma_{1,1, n}\right)$ on its boundary, see Figure 5. (By slightly perturbing $a_{n}$, the level line $u_{1, n}=a_{n}$ consists of a finite number of regular Jordan curves.) The boundary of $\Sigma_{1}$ consists of a Jordan curve $\alpha_{1}$ on which $u_{1, n}=a_{n}$, and one or several small convex curves $\pi\left(\gamma_{1, i, n}\right)$ with $i \leq m$, on which $u_{1, n}>a_{n}$. (The fact that $i \leq m$ can be ensured by taking the constant $C$ large enough.)

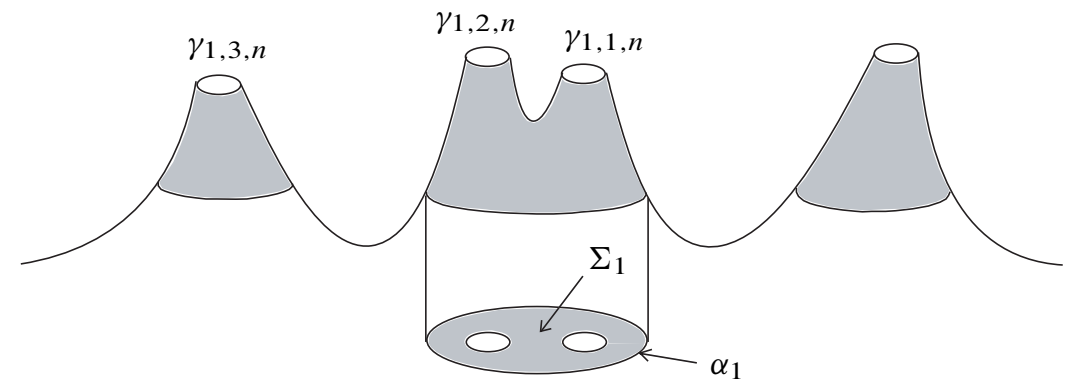

Figure 5. Definition of $\Sigma_{1}$ and $\alpha_{1}$, here $m=2$.

Let $\rho_{1}$ be the function which is equal to $\left|\nabla u_{1, n}\right|$ on $\Sigma_{1}$, and zero elsewhere. We first estimate the area $A\left(\rho_{1}\right)$ by the following interesting computation, writing $u=u_{1, n}$ and $v$ the unit exterior co-normal along $\partial \Sigma_{1}$ :

$$
\begin{aligned}
A\left(\rho_{1}\right) & =\iint_{\Sigma_{1}}|\nabla u|^{2} \leq \sqrt{2} \iint_{\Sigma_{1}} \frac{|\nabla u|^{2}}{\sqrt{1+|\nabla u|^{2}}} \\
& =\sqrt{2} \iint_{\Sigma_{1}} \operatorname{div}\left(\left(u-a_{n}\right) \frac{\nabla u}{\sqrt{1+|\nabla u|^{2}}}\right) \\
& =\sqrt{2} \int_{\partial \Sigma_{1}}\left(u-a_{n}\right) \frac{\frac{\partial u}{\partial v}}{\sqrt{1+|\nabla u|^{2}}} \\
& \leq 4 \pi \sum_{i} \frac{\eta_{i, n}-a_{n}}{\lambda_{1, n}} .
\end{aligned}
$$

On the first line we have used $|\nabla u| \leq 1$. On the second line we have used the minimal surface equation. On the third line, the divergence theorem. For the last 
line, we estimate each boundary term: the term along $\alpha_{1}$ vanishes since $u=a_{n}$. Along each small convex curve $\pi\left(\gamma_{1, i, n}\right)$, we have $u \leq \eta_{i, n}$ and $\frac{\partial u}{\partial v}>0$, so the integral can be estimated by the flux along this curve, which is close to $\frac{2 \pi}{\lambda_{i, n}} \leq \frac{2 \pi}{\lambda_{1, n}}$. The sum is on all indices $i$ such that $\pi\left(\gamma_{1, i, n}\right)$ lies on the boundary of $\Sigma_{1}$. There are at most $m$ terms. We do the same argument for the function $u_{2, n}$, considering the set $u_{2, n}>t_{n}-a_{n}$ and writing $\rho_{2}=\left|\nabla u_{2, n}\right|$, and we obtain

$$
A\left(\rho_{2}\right) \leq 4 \pi \sum_{i} \frac{t_{n}-\eta_{i, n}-a_{n}}{\lambda_{1, n}} .
$$

Adding the two estimates gives

$$
A\left(\rho_{1}\right)+A\left(\rho_{2}\right) \leq 4 \pi m \frac{t_{n}-2 a_{n}}{\lambda_{1, n}} .
$$

Let $\mathcal{A}$ be the annulus bounded by $\alpha_{1}$ and $\pi\left(\gamma_{1,1, n}\right)$. We consider the family $\Gamma$ of curves in $\mathcal{A}$ which connect the two boundary components. By definition of $L\left(\rho_{1}\right)$, there exists a curve $\gamma$ in $\Gamma$ such that $L_{\gamma}\left(\rho_{1}\right)$ is less than say $L\left(\rho_{1}\right)+\frac{1}{\lambda_{1, n}}$. If $\gamma$ happens to enter one of the small disks bounded by $\pi\left(\gamma_{1, i, n}\right)$ (with $2 \leq i \leq m$ ), then we replace the portion of $\gamma$ inside this disk (where $\rho_{1}=0$ ) by an arc on the boundary of the disk (where $\rho_{1}=|\nabla u|$ ). This increases $L\left(\rho_{1}\right)$ by an amount less than the flux along this curve. We may also assume that $\gamma$ enters each disk at most once by shunting all unnecessary circuits. This way, we obtain a curve $\gamma$ which stays inside $\Sigma_{1}$ and such that $L_{\gamma}\left(\rho_{1}\right) \leq L\left(\rho_{1}\right)+\frac{C^{\prime}}{\lambda_{1, n}}$ for some uniform constant $C^{\prime}$. Then we write

$$
\eta_{1, n}-a_{n}=\int_{\gamma} d u \leq \int_{\gamma}|\nabla u|=L_{\gamma}\left(\rho_{1}\right) \leq L\left(\rho_{1}\right)+\frac{C^{\prime}}{\lambda_{1, n}} .
$$

We do the same thing for $u_{2, n}$ and add the two estimates, we obtain

$$
t_{n}-2 a_{n} \leq L\left(\rho_{1}\right)+L\left(\rho_{2}\right)+\frac{2 C^{\prime}}{\lambda_{1, n}} \leq 2\left(L\left(\rho_{1}\right)+L\left(\rho_{2}\right)\right) .
$$

To obtain the last inequality, we observe that if $L\left(\rho_{1}\right)+L\left(\rho_{2}\right) \leq \frac{2 C^{\prime}}{\lambda_{1, n}}$, then $t_{n} \leq$ $\frac{2 C+4 C^{\prime}}{\lambda_{n}}$, but this is impossible since $t_{n} \gg \frac{1}{\lambda_{n}}$ by equation (6).

To estimate the modulus of the annulus $\mathcal{A}$, we observe that we can find a uniform $R$ such that $\mathcal{A}$ is contained in the annulus $D\left(p_{1, n}, R\right) \backslash D\left(p_{1, n}, \frac{1}{\lambda_{1, n}}\right)$. By monotonicity of the modulus, the modulus of $\mathcal{A}$ is bounded by $\frac{1}{2 \pi} \log \left(R \lambda_{1, n}\right)$, which we can safely bound by $\log \lambda_{1, n}$ for $n$ large enough. All this gives, using the definition of the modulus as an extremal length,

$$
\begin{aligned}
\left(t_{n}-2 a_{n}\right)^{2} & \leq 4\left(L\left(\rho_{1}\right)+L\left(\rho_{2}\right)\right)^{2} \leq 8\left(L\left(\rho_{1}\right)^{2}+L\left(\rho_{2}\right)^{2}\right) \\
& \leq 8 \log \left(\lambda_{1, n}\right)\left(A\left(\rho_{1}\right)+A\left(\rho_{2}\right)\right) \\
& \leq 32 \pi m\left(t_{n}-2 a_{n}\right) \frac{\log \lambda_{1, n}}{\lambda_{1, n}}
\end{aligned}
$$


It follows that

$$
t_{n} \leq 32 \pi m \frac{\log \lambda_{1, n}}{\lambda_{1, n}}+\frac{2 C}{\lambda_{n}} .
$$

This upper bound for $t_{n}$ is similar to (5), although the constant is not as good. Using the lower bound, equation (6), and arguing as in the last paragraph of the proof of Proposition 8 , we obtain that $c_{1} \geq \frac{\beta}{16 \pi m}>0$. This implies in particular that the point $p_{1}$ is interior by Proposition 10 .

Let $\delta_{n}=\min \left\{d\left(p_{1, n}, p_{j, n}\right): 2 \leq j \leq m\right\}$. Observe that $\delta_{n} \lambda_{1, n} \rightarrow \infty$. Passing to a subsequence and changing indices, we may assume that $\delta_{n}=d\left(p_{1, n}, p_{2, n}\right)$. We want to prove that $c_{2}>0$. Proposition 10 then implies that $p_{1} \neq p_{2}$, a contradiction.

Let $q_{n}$ be the middle point of $p_{1, n}, p_{2, n}$. Fix as before some $\alpha>1$ and $\beta<1$ close to 1 . Using the catenoidal barrier $\mathcal{C}\left(\frac{\beta}{\lambda_{1, n}}, \frac{\delta_{n}}{2}\right)$ as in the proof of Proposition 8 , we can estimate $\eta_{1, n}-u_{1, n}\left(q_{n}\right)$ and $u_{2, n}\left(q_{n}\right)-\eta_{1, n}$. Adding the two estimates gives the lower bound

$$
u_{2, n}\left(q_{n}\right)-u_{1, n}\left(q_{n}\right) \geq \frac{2 \beta}{\lambda_{1, n}} \operatorname{argcosh}\left(\frac{\delta_{n} \lambda_{1, n}}{2 \beta}\right)-\frac{C}{\lambda_{n}} \geq \frac{2 \beta}{\lambda_{1, n}} \log \left(\delta_{n} \lambda_{1, n}\right)-\frac{C^{\prime}}{\lambda_{n}} .
$$

Let $\varphi_{n}$ be the homothety of ratio $\mu_{n}=1 / \delta_{n}$ which maps $p_{1, n}$ to 0 . Let $\tilde{p}_{i, n}=$ $\varphi_{n}\left(p_{i, n}\right)$. Passing to a subsequence, $\lim \tilde{p}_{i, n}=\tilde{p}_{i}$ exists (possibly infinite), with $\tilde{p}_{1}=0$ and $\left|\tilde{p}_{2}\right|=1$. If all other $\tilde{p}_{j}$ are distinct from $\tilde{p}_{2}$, then there exists $\varepsilon \in(0,1)$ such that the disk $D\left(\tilde{p}_{2}, \varepsilon\right)$ contains no other point $\tilde{p}_{j}$. Going back to the original scale, we can use the catenoidal barrier $\ell\left(\frac{\alpha}{\lambda_{2, n}}, \delta_{n} \varepsilon\right)$ to estimate $\eta_{2, n}-u_{1, n}\left(a_{n}\right)$ and $u_{2, n}\left(a_{n}\right)-\eta_{2, n}$. Adding the two estimates gives the upper bound

$$
u_{2, n}\left(a_{n}\right)-u_{1, n}\left(a_{n}\right) \leq \frac{2 \alpha}{\lambda_{2, n}} \log \left(\delta_{n} \lambda_{2, n}\right)+\frac{C^{\prime}}{\lambda_{n}}
$$

for some uniform constant $C^{\prime}$. Combining the two estimates, we obtain after elementary operations

$$
\left(\beta-\alpha \frac{\lambda_{1, n}}{\lambda_{2, n}}\right) \log \left(\delta_{n} \lambda_{1, n}\right) \leq C^{\prime} \frac{\lambda_{1, n}}{\lambda_{n}}+\alpha \frac{\lambda_{1, n}}{\lambda_{2, n}} \log \left(\frac{\lambda_{2, n}}{\lambda_{1, n}}\right) .
$$

The right member has a finite limit (for the first term, this is because $c_{1}>0$ ). Since $\delta_{n} \lambda_{1, n} \rightarrow \infty$, we must have $\beta \leq \alpha \frac{\lambda_{1, n}}{\lambda_{2, n}}$ for $n$ large enough. Hence $\beta \frac{\lambda_{n}}{\lambda_{1, n}} \leq \alpha \frac{\lambda_{n}}{\lambda_{2, n}}$. Passing to the limit gives $\beta c_{1} \leq \alpha c_{2}$. Since we already know that $c_{1}>0$, we obtain $c_{2}>0$, hence by Proposition $10, p_{1} \neq p_{2}$, a contradiction. In case there are several points $\tilde{p}_{j}$ equal to $\tilde{p}_{2}$, we use instead the above extremal length argument to estimate $u_{2, n}\left(a_{n}\right)-u_{1, n}\left(a_{n}\right)$, and conclude again that $c_{2}>0$. This proves the proposition. 
Vol. 85 (2010) On minimal surfaces bounded by two convex curves in parallel planes

\section{References}

[1] L. V. Ahlfors, Lectures on quasiconformal mappings. Van Nostrand, Princeton, N.J., 1966. Zbl 0138.06002 MR 0200442

[2] C. Bandle and M. Flucher, Harmonic radius and concentration of energy; hyperbolic radius and Liouville's equation. SIAM Review 38 (2) (1996), 191-238. Zbl 0857.35034 MR 1391227

[3] J. Choe and M. Soret, Nonexistence of certain complete minimal surfaces with planar ends. Comment. Math. Helv. 75 (2000), 189-199. Zbl 0968.53008 MR 1760502

[4] T. Ekholm, B. White, and D. Wienholtz, Embeddedness of minimal surfaces with total

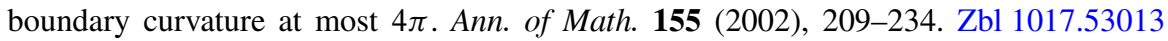
MR 1888799

[5] B. Gustafsson, On the convexity of a solution of Liouville's equation. Duke Math. J. 60 (2) (1990), 303-311. Zbl 0719.35002 MR 1047755

[6] O. Lehto and K. Virtanen, Quasiconformal mappings in the plane. Grundlehren Math. Wiss. 126, Springer-Verlag, Heidelberg 1973. Zbl 0267.30016 MR 0344463

[7] W. H. Meeks III and H. Rosenberg, The geometry of periodic minimal surfaces. Comment. Math. Helv. 68 (1993), 538-578. Zbl 0807.53049 MR 1241472

[8] W. H. Meeks III and B. White, Minimal surfaces bounded by convex curves in parallel planes. Comment. Math. Helv. 66 (1991), 263-278. Zbl 0731.53004 MR 1107841

[9] J. Pérez and A. Ros, Properly embedded minimal surfaces with finite total curvature. In The global theory of minimal surfaces in flat spaces (ed. by G. P. Pirola), Lecture Notes in Math. 1775, Springer-Verlag, Berlin 2002, 15-66. Zbl 1028.53005 MR 1901613

[10] A. Ros, Compactness of spaces of properly embedded minimal surfaces with finite total curvature. Indiana Univ. Math. J. 44 (1) (1995), 139-152. Zbl 0826.53005 MR MR1336435

[11] A. Ros, Embedded minimal surfaces: forces, topology and symmetries. Calc. Var. Partial Differential Equations 4 (1996), 469-496. Zbl 861.53008 MR 1402733

[12] R. Schoen, Uniqueness, symmetry, and embeddedness of minimal surfaces. J. Differential Geom. 18 (1983), 791-809. Zbl 0575.53037 MR 0730928

[13] M. Shiffman, On surfaces of stationary area bounded by two circles, or convex curves, in parallel planes. Ann. of Math. 63 (1956), 77-90. Zbl 0070.16803 MR 0074695

[14] B. White, Curvature estimates and compactness theorems in 3-manifolds for surfaces that are stationary for parametric elliptic functionals. Invent. Math. 88 (2) (1987), 243-256. Zbl 0615.53044 MR 0880951

Received January 15, 2008

Martin Traizet, Laboratoire de Mathématiques et Physique Théorique, Université de Tours, 37200 Tours, France

E-mail: martin.traizet@1mpt.univ-tours.fr 